\title{
Analyticity of Nekrasov Partition Functions
}

\author{
Giovanni Felder (D), Martin Müller-Lennert \\ Department of Mathematics, ETH Zurich, 8092 Zurich, Switzerland. \\ E-mail: felder@math.ethz.ch; m.muellerlennert@gmail.com
}

Received: 5 October 2017 / Accepted: 29 April 2018

Published online: 5 October 2018 - (C) The Author(s) 2018

\begin{abstract}
We prove that the $K$-theoretic Nekrasov instanton partition functions have a positive radius of convergence in the instanton counting parameter and are holomorphic functions of the Coulomb parameters in a suitable domain. We discuss the implications for the AGT correspondence and the analyticity of the norm of Gaiotto states for the deformed Virasoro algebra. The proof is based on random matrix techniques and relies on an integral representation of the partition function, due to Moore, Nekrasov, and Shatashvili, which we also prove.
\end{abstract}

\section{Contents}

1. Introduction . . . . . . . . . . . . . . . . . . . . . . 684

2. Nekrasov Partition Functions . . . . . . . . . . . . . . . . . . 686

2.1 A Nakajima quiver variety . . . . . . . . . . . . . . . . . 686

2.2 Notations for $K$-theory . . . . . . . . . . . . . . . . . . . . . . 687

2.3 Tangent spaces at fixed points . . . . . . . . . . . . . . 688

2.4 The tautological bundle . . . . . . . . . . . . . . . . 688

$2.5 K$-theoretic Nekrasov partition function . . . . . . . . . . . . . . 688

2.6 Integral representation and estimate . . . . . . . . . . . . . . . 689

2.7 Potential theory . . . . . . . . . . . . . . . . 693

2.8 Identification of coefficients and conclusion . . . . . . . . . . . 698

3. Generalization ..................... . 699

3.1 Different parameter ranges . . . . . . . . . . . . . . . . . . . 699

3.2 Similar types of gauge theory . . . . . . . . . . . . . . . . . . 699

4. Specialization: Norm of Deformed Gaiotto States . . . . . . . . . . . . . . 699

4.1 Definition . . . . . . . . . . . . . . . . . . . 699

4.2 Finiteness of norm . . . . . . . . . . . . . . . 701

5. Open Problems . . . . . . . . . . . . . . . . . 701

5.1 Homological version of Nekrasov partition function . . . . . . . . . 701 
5.2 Conformal blocks . . . . . . . . . . . . . . . 703

References . . . . . . . . . . . . . . . 717

\section{Introduction}

The purpose of this paper is to study the analytic properties of the Nekrasov instanton partition function. In $\mathcal{N}=2$ supersymmetric gauge theory in four and five dimensions, Nekrasov's instanton partition function [18] plays the role of a basic building block. In the physical interpretation, the instanton partition function is the non-perturbative contribution of instantons to a gauge theory in an $\Omega$-background. Mathematically it is the generating function of integrals of torus equivariant cohomology classes $(K$ theory classes in the five dimensional theory) on the moduli space of framed torsion free sheaves with fixed rank on the complex projective plane. The torus is a product of a two-dimensional torus acting on the projective plane and a torus acting on the framing. The parameters $\epsilon_{1}, \epsilon_{2}$ of the $\Omega$-background are the equivariant parameters of the twodimensional torus in the mathematical description and serve as an infrared regulator. One early success of the theory $[18,19]$ was a microscopic justification of the SeibergWitten formula [22] for the prepotential of the low energy effective theory, which arises in the limit $\epsilon_{1}, \epsilon_{2} \rightarrow 0$. The Nekrasov instanton partition function is a power series in a complex variable $\mathfrak{q}$ parametrizing the strength of the interaction in gauge theory. The coefficient of $\mathfrak{q}^{n}$ is the contribution of instanton number $n$ to the partition function. The simplest case of a Nekrasov partition function appears in the pure $\mathcal{N}=2$ supersymmetric Yang-Mills theory with gauge group $U(r)$ on $\mathbb{R}^{4} \times S^{1}$. It is given as a sum over $r$-tuples $\vec{Y}=\left(Y_{i}\right)_{i=1}^{r}$ of Young diagrams of total size $|\vec{Y}|$ :

$$
\begin{aligned}
Z\left(\epsilon_{1}, \epsilon_{2}, a, \mathfrak{q}, \lambda\right) & =\sum_{\vec{Y}} \mathfrak{q}^{|\vec{Y}|} \prod_{\alpha, \beta=1}^{r} \prod_{b \in Y_{\alpha}} \frac{(\lambda / 2)^{2}}{\sinh \left(\frac{\lambda}{2} E_{\alpha \beta}(b)\right) \sinh \left(\frac{\lambda}{2}\left(\epsilon_{1}+\epsilon_{2}-E_{\alpha \beta}(b)\right)\right)}, \\
E_{\alpha \beta}(b) & =a_{\alpha}-a_{\beta}-l_{Y_{\beta}}(b) \epsilon_{1}+\left(a_{Y_{\alpha}}(b)+1\right) \epsilon_{2} .
\end{aligned}
$$

Here $\lambda$ is the circumference of the circle $S^{1}$ and $a=\left(a_{1}, \ldots, a_{r}\right)$ belongs to the Lie algebra of $U(r)$ and parametrizes boundary conditions of scalar fields in the $\mathcal{N}=2$ vector multiplet. The arm length $a_{Y}(b)$ of a box $b$ is the number of boxes in the Young diagram $Y$ to the right of $b$; the leg length $l_{Y}(b)$ is the (possibly negative) number of boxes in $Y$ below $b$. The four dimensional theory on $\mathbb{R}^{4}$ arises in the limit $\lambda \rightarrow 0$ and amounts to replacing $\sinh (\lambda x) / \lambda$ by $x$ and $\mathfrak{q}=\Lambda^{2 r}$ is related to the dynamical mass scale $\Lambda$ of the gauge theory. In the mathematical description, we view $\mathbb{R}^{4}$ as $\mathbb{C}^{2}$ with its action of $\left(\mathbb{C}^{\times}\right)^{2}$ and embed it in $\mathbb{C P}^{2}$ by adding a line at infinity $\ell_{\infty} \cong \mathbb{C P}^{1}$. Let $\mathcal{M}(r, n)$ be the moduli space of torsion free sheaves on $\mathbb{C P}^{2}$ of rank $r$ and second Chern class $n$ with a framing, i.e., a trivialization on $\ell_{\infty}$. It is a smooth algebraic variety of dimension $2 n r$. The action of the group $\left(\mathbb{C}^{\times}\right)^{2}$ lifts to an action on $\mathcal{M}(r, n)$. Moreover $G L_{r}$, and in particular its Cartan torus $T=\left(\mathbb{C}^{\times}\right)^{r}$ acts on $\mathcal{M}(r, n)$ by changing the framing. Therefore we have an action of $\tilde{T}=\left(\mathbb{C}^{\times}\right)^{2} \times T$ on $\mathcal{M}(r, n)$. For a semisimple $\tilde{T}$-module $V$ with finite dimensional weight spaces $V_{\chi}$, we denote by $\operatorname{ch}(V)=\sum_{\chi} e^{\chi} \operatorname{dim} V_{\chi}$ its formal character in the completed group ring of the weight lattice. It is a formal series in the weight variables $q_{1}, q_{2}$ for the $\left(\mathbb{C}^{\times}\right)^{2}$-action and $u_{1}, \ldots, u_{r}$ for the $T$-action. Then

$$
Z\left(\epsilon_{1}, \epsilon_{2}, a, \mathfrak{q}, \lambda\right)=\sum_{n=0}^{\infty} z^{n} \sum_{i=1}^{2 n r}(-1)^{i} \operatorname{ch} H^{i}(\mathcal{M}(r, n), \mathcal{O}), \quad z=\mathfrak{q} \lambda^{2 r} e^{-\lambda\left(\epsilon_{1}+\epsilon_{2}\right) r / 2},
$$


is the pushforward of the $\tilde{T}$-equivariant $K$-theory class of the structure sheaf by the map to a point, with the identification

$$
q_{1}=e^{-\lambda \epsilon_{1}} . \quad q_{2}=e^{-\lambda \epsilon_{2}}, \quad u_{\alpha}=e^{-\lambda a_{\alpha}}, \quad \alpha=1, \ldots, r .
$$

The combinatorial formula above is obtained by localization to fixed points. There are many variants and generalizations of the Nekrasov partition functions. In this paper we focus on the case where one includes matter fields in the gauge theory, which corresponds to replacing the structure sheaf by more general $K$-theory classes in the mathematical description.

The absolute value squared $|Z|^{2}$ of the Nekrasov partition function, in the limit $\lambda \rightarrow 0$, also appears in the integrand of Pestun's formula for gauge theory on the round sphere $S^{4}$ [21] and in its extension to $S^{4}$ with an ellipsoid metric parametrized by $\epsilon_{1}, \epsilon_{2}>0$ [11]. For $\lambda>0$ this formula extends to $S^{4} \times S^{1}$ and other compact manifolds, see [20] for a review. For these reasons it is important to understand the convergence properties of the formal power series defining the partition function. In this paper, we prove that the power series has a positive radius of convergence if $\lambda>0$ and in a suitable range of parameters. For example, in the case of pure gauge theory, we show that if $\lambda>0$ and $\epsilon_{1}, \epsilon_{2}>0$, the series (1) converges for all $z$ in the unit disk to an analytic function of the Coulomb parameters $a_{i}$ in a neighbourhood of the imaginary axis, see Theorem 5 for the general result. Note that for this range of parameters a direct estimation of the sum over partitions is problematic because of small denominators. In fact, the individual terms are not defined if $\epsilon_{1} / \epsilon_{2} \in \pi \mathbb{Q}$ and have arbitrarily small denominators otherwise. The situation is different in the case $\epsilon_{1}<0<\epsilon_{2}$ (which we do not consider) where a positive radius of convergence may be obtained by direct estimates on individual terms for generic $a_{i}$. Our proof applies to the case $\epsilon_{1}, \epsilon_{2}>0$ and also $\epsilon_{1}=\bar{\epsilon}_{2}$ not imaginary, and relies on an integral representation of the coefficients of $Z$ which resembles a unitary random matrix integral. This integral representation is actually the form in which this type of partition functions first appeared in the literature, as a regularized volume of instanton moduli spaces, see [14, , Section 6]. We estimate it using methods of random matrix theory and an explicit formula from representation homology [4]. The combinatorial formula for $Z$ above is the sum of residues at certain poles of the integrand. As the choice of integration cycle is essential for the estimate, we carefully prove that the integral formula with the correct integration cycle is equal to the combinatorial formula. This requires showing that certain apparent residues actually cancel out. The limit $\lambda \rightarrow 0$ to the four dimensional theory is subtle and appears to require a different approach; we hope to return to this problem in the future.

Another reason for the interest in the radius of convergence of the power series $Z$ comes from conformal field theory in 2 dimensions through the Alday-GaiottoTachikawa (AGT) correspondence [2]. According to this correspondence, which was verified in a number of cases, the instanton partition functions of $\mathcal{N}=2$ supersymmetric gauge theories with suitable matter fields are equal, up to a known scalar factor, to conformal blocks of $W$-algebras. For example, for $r=2$ and $\lambda=0$ they are related to the four point conformal blocks of the Virasoro algebra is defined as a matrix element of products of certain intertwining operators (primary fields). The parameters are the central charge $c=13+6 b^{2}+6 b^{-2}$ with $\epsilon_{1}=b=\epsilon_{2}^{-1}$ and the highest weights of four Virasoro representations, related to masses of matter fields. A priori the four point conformal block is a formal power series in the cross ratio $\mathfrak{q}$ of the four points. Except in the special cases of degenerate representations, occurring in minimal models at $c<1$, where they are solutions of differential equations of hypergeometric type, giving full control on the 
radius of convergence and analytic continuation, representation theory does not seem to give information on the convergence of the power series. This is particularly relevant for the unitary representations of the Virasoro algebra with $c>1$, arising in Liouville theory. We treat both the weakly coupled Liouville theory with $c \geq 25$ and $\epsilon_{1}, \epsilon_{2}>0$ and the strongly coupled range $1<c \leq 25$, with $\epsilon_{1}=\bar{\epsilon}_{2}$ on the unit circle. The limiting case $c=1\left(\epsilon_{1}=i=-\epsilon_{2}\right)$ was considered for $\lambda=0$ in [12]. The case $\epsilon_{1}=-\epsilon_{2}$ for $\lambda \neq 0$ was recently considered in [5]. In cases where the AGT correspondence is understood, our estimates on the gauge theory side imply the convergence of conformal blocks. We make this explicit in the mathematically well-understood case of the norm of Gaiotto states for the $q$-deformed Virasoro algebra. Gaiotto states [9] are Whittaker vectors in completions of Verma modules of $W$-algebras and their deformations. In the case of the Virasoro algebra a Gaiotto state is a formal power series in the eigenvalue of the generator $L_{1}$. The squared norm of a Gaiotto state can be understood as suitable limits of a conformal block and corresponds via the AGT correspondence to the Nekrasov partition function of the pure Yang-Mills theory. As an application of our result we prove that for a suitable range of parameters the squared norm of the Gaiotto state for the two-parameter deformation of the Virasoro algebra is a holomorphic function of the eigenvalue with a convergence radius that converges to infinity in the $\lambda \rightarrow 0$ limit.

This agrees with the findings of Its et al. [12] and Bershtein et al. [5]. They consider the norm of the Gaiotto state in the case $\lambda=0$ and $\lambda>0$, respectively. Moreover, they require $\epsilon_{1}+\epsilon_{2}=0$, which is different from our setting. In their respective setups, the Plancherel measure on partitions appears and allows for a direct estimate, which proves analyticity of the norm of the Gaiotto state on the whole complex plane.

The paper is organized as follows: in Sect. 2 we review the mathematical definition of the Nekrasov partition function with matter fields by introducing $M(r, n)$ as a special case of a Nakajima quiver variety via the ADHM construction and explain the localization formula leading to the combinatorial expression for $Z$. We then introduce the integral representation of $Z$ and compute the large $n$ behaviour of the coefficient of $\mathfrak{q}^{n}$ using methods of random matrix theory and estimate the radius of convergence. In Sect. 3 we discuss how our method can be generalized to more general gauge theory, including the $\mathcal{N}=2^{*}$ theory. In Sect. 4 we present the application to the norm of Gaiotto states for the deformed Virasoro algebra. In Sect. 5 we discuss some of the problems that are left open. The Appendix contains the proof of the integral representation of the partition function.

\section{Nekrasov Partition Functions}

In this section, we define the $K$-theoretic Nekrasov partition function as discussed in [16]. It is defined as a formal power series. Our aim is to show that for certain parameter ranges, the series converges.

2.1. A Nakajima quiver variety. We follow the exposition in $[15,16]$. Fix a positive integer $r$. The Nekrasov partition function is a generating functions for certain $K$-theory classes computed from a sequence of so-called Nakajima quiver varieties $M(r, n)$, where $n=0,1,2, \ldots$ They are constructed as follows: Set $V=\mathbb{C}^{n}$ and $W=\mathbb{C}^{r}$. We define

$$
\begin{aligned}
M(r, n)=\{ & \left(B_{1}, B_{2}, i, j\right) \in \operatorname{End}(V) \times \operatorname{End}(V) \times \operatorname{Hom}(W, V) \times \operatorname{Hom}(V, W): \\
& {\left[B_{1}, B_{2}\right]+i j=0 \text { and there does not exist a proper subspace } S \subset V }
\end{aligned}
$$




$$
\text { such that } \left.B_{1}(S), B_{2}(S) \subset S \text { and } i(W) \subset S\right\} / G L(V) \text {, }
$$

where an element $g \in G L(V)$ acts on $\left(B_{1}, B_{2}, i, j\right)$ via

$$
g \cdot\left(B_{1}, B_{2}, i, j\right)=\left(g B_{1} g^{-1}, g B_{2} g^{-1}, g i, j g^{-1}\right) .
$$

The space $M(r, n)$ is a nonsingular algebraic variety of dimension $2 n r$.

Let $T \subset G L_{r}(\mathbb{C})$ be the maximal torus consisting of diagonal matrices. The group $\tilde{T}=T \times \mathbb{C}^{*} \times \mathbb{C}^{*}$ acts on $M(r, n)$. This action is induced ${ }^{1}$ by

$$
\left(B_{1}, B_{2}, i, j\right) \cdot\left(t_{1}, t_{2}, e_{1}, \ldots, e_{r}\right)=\left(t_{1} B_{1}, t_{2} B_{2}, i e, t_{1} t_{2} e^{-1} j\right),
$$

where $\left(t_{1}, t_{2}, e_{1}, \ldots, e_{r}\right) \in \tilde{T}$ and $e$ is the diagonal matrix with entries $e_{1}, \ldots, e_{r}$.

We want to consider $\tilde{T}$-equivariant $K$-theory on $M(r, n)$. By the localization principle, we first want a description of the fixed points of the $\tilde{T}$-action. In order to describe the fixed points of this action, we use partitions. First we fix our conventions: We write $l(Y)=l$ for the length of the partition $Y=(Y(1), \ldots, Y(l))$ and $|Y|$ for its size $Y(1)+\cdots+Y(l)$. We use the English convention to draw the Young diagram corresponding to the partitions. For example the partition $Y=(5,3,2)$ of size 10 has length $l(Y)=3$ and its Young diagram is given by

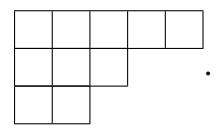

In the Young diagram, the row index $x$ increases as we go south and the column index $y$ increases as we go east. In the following we identify the partition $Y$ with its Young diagram. In particular, we write $Y=\{(x, y): 1 \leq x \leq l(Y), 1 \leq y \leq Y(x)\}$.

The fixed point set $M(r, n)^{\tilde{T}}$ is a discrete set whose points $I_{\vec{Y}}$ are indexed by $r$-tuples $\vec{Y}=\left(Y_{1}, \ldots, Y_{r}\right)$ of partitions with total size $|\vec{Y}|:=\left|Y_{1}\right|+\cdots+\left|Y_{r}\right|=n$. We denote the corresponding inclusion maps as follows:

$$
\iota: M(r, n)^{\tilde{T}} \rightarrow M(r, n), \quad \iota_{\vec{Y}}:\left\{I_{\vec{Y}}\right\} \rightarrow M(r, n), \quad|\vec{Y}|=n .
$$

2.2. Notations for $K$-theory. Let $t_{i}$ be the $\tilde{T}$-character given by $\left(t_{1}, t_{2}, e_{1}, \ldots, e_{r}\right) \mapsto t_{i}$. Let $e_{\alpha}$ be the $\tilde{T}$-character given by $\left(t_{1}, t_{2}, e_{1}, \ldots, e_{r}\right) \mapsto e_{\alpha}$. All the $\tilde{T}$-equivariant $K$ theory groups $K^{\tilde{T}}(-)$ are modules for the ring

$$
R(\tilde{T}):=K^{\tilde{T}}(p t) \cong \mathbb{Z}\left[t_{1}^{ \pm 1}, t_{2}^{ \pm 1}, e_{1}^{ \pm 1}, \ldots, e_{r}^{ \pm 1}\right] .
$$

Let $\mathcal{R} \cong \mathbb{Q}\left(t_{1}, t_{2}, e_{1}, \ldots, e_{r}\right)$ denote its quotient field. The maps $\iota$ and $\iota_{\vec{Y}}$ all define pushforwards in K-theory. The Thomason localization theorem in K-theory says that

$$
\iota_{*}: K^{\tilde{T}}\left(M(r, n)^{\tilde{T}}\right) \rightarrow K^{\tilde{T}}(M(r, n))
$$

becomes an isomorphism after localization, i.e. tensoring with $\mathcal{R}$. We write

$$
K_{\text {loc }}^{\tilde{T}}(-)=K^{\tilde{T}}(-) \otimes_{R(\tilde{T})} \mathcal{R} .
$$

\footnotetext{
1 Note our convention differs from the one used in [15] by $e_{\alpha} \mapsto e_{\alpha}^{-1}$.
} 
2.3. Tangent spaces at fixed points. We will need a description of the tangent space $T_{\vec{Y}} M(r, n)$ at a fixed point $I_{\vec{Y}}$ as a $\tilde{T}$-module. It is given by [15, Theorem 2.11]

$$
\begin{aligned}
T_{\vec{Y}} M(r, n) & =\sum_{\alpha, \beta=1}^{r} N_{\alpha, \beta}\left(t_{1}, t_{2}\right) \in K^{\tilde{T}}(p t), \\
N_{\alpha, \beta}\left(t_{1}, t_{2}\right) & =e_{\alpha} e_{\beta}^{-1}\left(\sum_{s \in Y_{\alpha}} t_{1}^{-l_{Y_{\beta}}(s)} t_{2}^{a_{Y_{\alpha}}(s)+1}+\sum_{t \in Y_{\beta}} t_{1}^{l_{Y_{\alpha}}(t)+1} t_{2}^{-a_{Y_{\beta}}(t)}\right) .
\end{aligned}
$$

Here we use the arm length $a_{Y}(s)=Y(x)-y$ and leg length $l_{Y}(s)=Y^{T}(y)-x$ of a box $s=(x, y) \in \mathbb{Z}^{2}$ with respect to the partition $Y$. The symbol $Y^{T}$ denotes the transpose of the partition $Y$. Note that box $s$ does not have to belong to the partition $Y$, hence arm length and leg length can be negative.

2.4. The tautological bundle. In order to include theories with massive matter, we introduce formal parameters $\vec{b}=\left(b_{1}, \ldots, b_{s}\right)$. The tautological bundle $\mathcal{V}$ is a vector bundle over $M(r, n)$ of rank $n$ whose fiber is $V$, see e.g. [17]. Let $c_{0}, \ldots, c_{n} \in K_{\text {loc }}^{\tilde{T}}(M(r, n))$ denote its Chern classes. Consider the polynomial $b(z)=\left(z-b_{1}\right) \cdots\left(z-b_{s}\right)$. Construct the element

$$
\kappa_{n}\left(b_{1}, \ldots, b_{s}\right):=\left.b\left(z_{1}\right) \cdots b\left(z_{n}\right)\right|_{\sigma_{j}=c_{j}} \in K_{\mathrm{loc}}^{\tilde{T}}(M(r, n))\left[b_{1}, \ldots, b_{s}\right]
$$

by expressing the symmetric polynomial $b\left(z_{1}\right) \cdots b\left(z_{n}\right)$ as a polynomial in the elementary symmetric functions $\sigma_{0}, \ldots, \sigma_{n}$ and replacing each $\sigma_{j}$ by the Chern class $c_{j}$.

2.5. K-theoretic Nekrasov partition function. We now define the Nekrasov partition function arising in the K-theory of the moduli space of instantons. Let $\left(\epsilon_{1}, \epsilon_{2}, a_{1}, \ldots, a_{r}\right)$ be coordinates on the Lie algebra of $\tilde{T}$ and $\lambda$ be a parameter such that $t_{j}=e^{\lambda \epsilon_{j}}$ and $e_{\alpha}=e^{\lambda a_{\alpha}}$. Write $b_{m}=e^{\lambda w_{m}}$. The Nekrasov partition function is defined as a formal power series

$$
Z\left(\epsilon_{1}, \epsilon_{2}, \vec{a} ; \vec{w} ; \mathfrak{q}, \lambda\right)=\sum_{n \geq 0}\left(\mathfrak{q} \lambda^{2 r-s} e^{-r \lambda\left(\epsilon_{1}+\epsilon_{2}\right) / 2}\right)^{n} Z_{n}\left(\epsilon_{1}, \epsilon_{2}, \vec{a} ; \vec{w} ; \lambda\right),
$$

with coefficients

$$
\begin{aligned}
Z_{n}\left(\epsilon_{1}, \epsilon_{2}, \vec{a} ; \vec{w} ; \lambda\right) & =\sum_{i=0}^{2 n r}(-1)^{i} \operatorname{ch} H^{i}\left(M(r, n), \kappa_{n}\left(b_{1}, \ldots, b_{s}\right)\right) \\
& =\sum_{|\vec{Y}|=n}\left(\iota_{*}\right)^{-1}\left(\kappa_{n}\left(b_{1}, \ldots, b_{s}\right)\right) \in \mathcal{R}\left[b_{1}, \ldots, b_{s}\right] .
\end{aligned}
$$

Here $\sum_{|\vec{Y}|=n}$ denotes the summation map

$$
K_{\mathrm{loc}}^{\tilde{T}}\left(M(r, n)^{\tilde{T}}\right)\left[b_{1}, \ldots, b_{s}\right]=\bigoplus_{|\vec{Y}|=n} \mathcal{R}\left[b_{1}, \ldots, b_{s}\right] \rightarrow \mathcal{R}\left[b_{1}, \ldots, b_{s}\right]
$$


The fixed point theorem says that after localization we have

$$
\left(\iota_{*}\right)^{-1}=\sum_{|\vec{Y}|=n} \frac{\iota_{\vec{Y}}^{*}}{\Lambda_{-1} T_{\vec{Y}} M(r, n)},
$$

where $\Lambda_{-1}$ denotes the alternating sum of exterior powers. Combining Eq. (4) with the description (2) of the tangent space at a fixed point to compute $\Lambda_{-1} T_{\vec{Y}} M(r, n)$, one gets

$$
Z_{n}\left(\epsilon_{1}, \epsilon_{2}, \vec{a} ; \vec{w} ; \lambda\right)=\sum_{|\vec{Y}|=n} \frac{\iota_{\vec{Y}}^{*}\left(\kappa_{n}\left(b_{1}, \ldots, b_{s}\right)\right)}{\prod_{\alpha, \beta=1}^{r} n_{\alpha, \beta}^{\vec{Y}}\left(\epsilon_{1}, \epsilon_{2}, \vec{a} ; \lambda\right)},
$$

where

$$
\begin{aligned}
n_{\alpha, \beta}^{\vec{Y}}\left(\epsilon_{1}, \epsilon_{2}, \vec{a} ; \lambda\right)= & \prod_{s \in Y_{\alpha}}\left(1-e^{-\lambda\left(-l_{Y_{\beta}}(s) \epsilon_{1}+\left(a_{Y_{\alpha}}(s)+1\right) \epsilon_{2}+a_{\alpha}-a_{\beta}\right)}\right) \\
& \prod_{t \in Y_{\beta}}\left(1-e^{-\lambda\left(\left(l_{Y_{\alpha}}(t)+1\right) \epsilon_{1}-a_{Y_{\beta}}(t) \epsilon_{2}+a_{\alpha}-a_{\beta}\right)}\right) .
\end{aligned}
$$

For $s=0$, i.e. $b(z)=1$, we have $\kappa_{n}(\emptyset)=\mathcal{O}$, the $K$-theory class corresponding to the structure sheaf on $M(r, n)$. This corresponds to pure Yang Mills theory and the numerator in Eq. (5) equals one. For general $\vec{b}=\left(b_{1}, \ldots, b_{s}\right)$ and a fixed point $I_{\vec{Y}}$, we have [17, equation (2.27)]

$$
\iota_{\vec{Y}}^{*}\left(\kappa_{n}\left(b_{1}, \ldots, b_{s}\right)\right)=\prod_{\alpha=1}^{r} \prod_{(x, y) \in Y_{\alpha}} \prod_{m=1}^{s}\left(e^{-\lambda\left(a_{\alpha}+(x-1) \epsilon_{1}+(y-1) \epsilon_{2}\right)}-b_{m}\right) .
$$

Our aim is to estimate the general form of the coefficients (5) in order to prove convergence of the $K$-theoretic partition function. To do so, we use an integral representation for the coefficients $Z_{n}\left(\epsilon_{1}, \epsilon_{2}, \vec{a}, \vec{w} ; \lambda\right)$.

2.6. Integral representation and estimate. In this section we define an integral representation for the coefficients of the Nekrasov partition function. We will use this representation to estimate the coefficients.

2.6.1. Definition of the integral. Let $q_{1}$ and $q_{2}$ be a pair of complex numbers in the open unit disk. Assume that either $q_{1}=\overline{q_{2}}$ or $q_{1}, q_{2} \in(0,1)$. Note that in either case $q_{1} q_{2}=\left|q_{1} q_{2}\right| \in(0,1)$. Later these numbers will be identified with exponential functions of $\epsilon_{1}, \epsilon_{2}$.

Fix $r \geq 1$. Let $\vec{u}=\left(u_{1}, \ldots, u_{r}\right)$ be a vector of complex numbers such that

$$
\left|q_{i}\right| \max _{\alpha=1, \ldots, r}\left|u_{\alpha}\right|<\min _{\alpha=1, \ldots, r}\left|u_{\alpha}\right|, \quad \forall i=1,2 .
$$

Let $\vec{p}=\left(p_{1}, \ldots, p_{s}\right)$ be another vector of complex numbers. The condition on $\left|u_{\alpha}\right|$ ensures that we can pick $\rho>0$ with

$$
\left|u_{\alpha}\right|<\rho<\left|q_{i}\right|^{-1}\left|u_{\alpha}\right| \quad \forall \alpha=1, \ldots, r, \forall i=1,2 .
$$


Let $C_{\rho} \subset \mathbb{C}$ be the set of complex numbers of modulus $\rho$. We define

$$
\begin{aligned}
\mathcal{Z}_{n}(\vec{u} ; \vec{p})= & \frac{1}{n !}\left(\frac{1-q_{1} q_{2}}{\left(1-q_{1}\right)\left(1-q_{2}\right)}\right)^{n} \\
& \int_{C_{\rho}^{n}} \prod_{j=1}^{n} \frac{d z_{j}}{2 \pi i z_{j}} \prod_{j=1}^{n} \prod_{m=1}^{s}\left(z_{j}-p_{m}\right) \mathcal{I}\left(z_{1}, \ldots, z_{n} ; \vec{u}\right),
\end{aligned}
$$

where the integrand contains the symmetric function

$$
\mathcal{I}\left(z_{1}, \ldots, z_{n} ; \vec{u}\right)=\prod_{j=1}^{n} \prod_{\alpha=1}^{r} \frac{-u_{\alpha} z_{j}}{\left(z_{j}-u_{\alpha}\right)\left(q_{1} q_{2} z_{j}-u_{\alpha}\right)} \prod_{1 \leq j \neq k \leq n} \frac{\left(z_{j}-z_{k}\right)\left(z_{j}-q_{1} q_{2} z_{k}\right)}{\left(z_{j}-q_{1} z_{k}\right)\left(z_{j}-q_{2} z_{k}\right)}
$$

In the following, we evaluate the coefficients $\mathcal{Z}_{n}(\vec{u} ; \vec{p})$ using residue calculus and estimate them. We then identify them with the coefficients $Z_{n}$ of the $K$-theoretic partition function up to a change of variables in Sect. 2.8.

2.6.2. Evaluation of the integral. We evaluate the integral in $\mathcal{Z}_{n}(\vec{u} ; \vec{p})$ using residue calculus. The residues are indexed by $r$-tuples $\vec{Y}$ of partitions $Y_{\alpha}$ with total size $|\vec{Y}|=$ $\left|Y_{1}\right|+\cdots+\left|Y_{r}\right|=n$. We again identify a partition with its Young diagram. For a box $s=(x, y) \in Y_{\alpha}$ we define

$$
z_{s}^{\alpha}=z_{x, y}^{\alpha}=u_{\alpha} q_{1}^{x-1} q_{2}^{y-1}
$$

Theorem 1. Under the assumption

$$
\begin{array}{ll}
u_{\alpha} u_{\beta}^{-1} \neq q_{1}^{x} q_{2}^{y}, & \forall x, y \in\{-n, \ldots, n\}, \quad \forall \alpha \neq \beta \in\{1, \ldots, r\}, \\
q_{1}^{x} \neq q_{2}^{y+1}, \quad q_{1}^{x+1} \neq q_{2}^{y}, & \forall x, y \in\{0, \ldots, n-1\},
\end{array}
$$

the value of $\mathcal{Z}_{n}(\vec{u} ; \vec{p})$ is given as a sum over $r$-tuples of partitions of total size $n$ in two equivalent ways:

$$
\begin{aligned}
\mathcal{Z}_{n}(\vec{u} ; \vec{p}) & =\sum_{|\vec{Y}|=n} \frac{\prod_{\alpha=1}^{r} \prod_{s \in Y_{\alpha}} \prod_{m=1}^{s}\left(z_{s}^{\alpha}-p_{m}\right)}{N_{\alpha, \beta}^{\vec{Y}}\left(q_{1}, q_{2}, \vec{u}\right)}, \\
N_{\alpha, \beta}^{\vec{Y}}\left(q_{1}, q_{2}, \vec{u}\right) & =\prod_{s \in Y_{\alpha}}\left(1-\frac{u_{\alpha}}{u_{\beta}} q_{1}^{l_{Y_{\alpha}}(s)+1} q_{2}^{-a_{Y_{\beta}}(s)}\right) \prod_{t \in Y_{\beta}}\left(1-\frac{u_{\alpha}}{u_{\beta}} q_{1}^{-l_{Y_{\beta}}(t)} q_{2}^{a_{Y_{\alpha}}(t)+1}\right) .
\end{aligned}
$$

Alternatively,

$$
\begin{aligned}
\mathcal{Z}_{n}(\vec{u} ; \vec{p}) & =\sum_{|\vec{Y}|=n} \frac{\prod_{\alpha=1}^{r} \prod_{s \in Y_{\alpha}} \prod_{m=1}^{s}\left(z_{s}^{\alpha}-p_{m}\right)}{M_{\alpha, \beta}^{\vec{Y}}\left(q_{1}, q_{2}, \vec{u}\right)}, \\
M_{\alpha, \beta}^{\vec{Y}}\left(q_{1}, q_{2}, \vec{u}\right) & =\prod_{s \in Y_{\alpha}}\left(1-\frac{u_{\alpha}}{u_{\beta}} q_{1}^{-l_{Y_{\beta}}(s)} q_{2}^{a_{Y_{\alpha}}(s)+1}\right) \prod_{t \in Y_{\beta}}\left(1-\frac{u_{\alpha}}{u_{\beta}} q_{1}^{l_{Y_{\alpha}}(t)+1} q_{2}^{-a_{Y_{\beta}}(t)}\right) .
\end{aligned}
$$


The proof is of this theorem is technical. In the literature, several arguments for the validity of this or similar formulae have been given, see $[8,10,17,18,24]$. They are based on taking the iterated residues at $z_{x, y}^{\alpha},(x, y) \in Y_{\alpha}, \alpha=1, \ldots, r$. However, the integrand also has further poles and it is a nontrivial fact, that we prove in this paper, that the residues at those poles cancel. The proof of Theorem 1 is postponed to Appendix A.

Remark 1 . The assumption (11) is necessary to ensure that all terms $\mathcal{Z}_{\vec{Y}}(\vec{u} ; \vec{p})$ are welldefined. If it is violated, some residues might not be simple residues anymore and consequently some $\mathcal{Z}_{\vec{Y}}(\vec{u} ; \vec{p})$ might be infinite. However, their sum $\mathcal{Z}_{n}(\vec{u} ; \vec{p})$ is still well-defined, as the integral in Eq. (8) is.

2.6.3. Estimate for the integral. In this section we apply potential theory to estimate the coefficients $\mathcal{Z}_{n}(\vec{u} ; \vec{p})$ in the integral form given by Eq. (8) in the limit of large $n$.

Theorem 2. We have

$$
\limsup _{n \rightarrow \infty}\left|\mathcal{Z}_{n}(\vec{u} ; \vec{p})\right|^{\frac{1}{n}} \leq \prod_{m=1}^{s} \max \left\{\left|p_{m}\right|,\left|u_{1}\right|, \ldots,\left|u_{r}\right|\right\} .
$$

We prove this by comparing the growth of $\mathcal{Z}_{n}(\vec{u} ; \vec{p})$ to the growth of the coefficients

$$
a_{n}=\frac{1}{n !}\left(\frac{1-q_{1} q_{2}}{\left(1-q_{1}\right)\left(1-q_{2}\right)}\right)^{n} \int_{C_{1}^{n}} \prod_{j=1}^{n} \frac{d z_{j}}{2 \pi i z_{j}} \prod_{j \neq k} \frac{\left(z_{j}-z_{k}\right)\left(z_{j}-q_{1} q_{2} z_{k}\right)}{\left(z_{j}-q_{1} z_{k}\right)\left(z_{j}-q_{2} z_{k}\right)} .
$$

We also want to introduce the language of potential theory. Let $\mathbb{T}=\mathbb{R} / 2 \pi \mathbb{Z}$ be the torus. Define $f: \mathbb{T} \rightarrow \mathbb{R} \cup\{\infty\}$ by

$$
f(\theta):=-\log \frac{\left|e^{i \theta}-1\right|\left|e^{i \theta}-q_{1} q_{2}\right|}{\left|e^{i \theta}-q_{1}\right|\left|e^{i \theta}-q_{2}\right|} .
$$

For each $n \in \mathbb{N}$, we define a probability measure on $\mathbb{T}^{n}$ by

$$
P_{n}(\theta) d \theta=\frac{1}{Z_{n}} e^{-\sum_{j \neq k} f\left(\theta_{k}-\theta_{j}\right)} d \theta, \quad d \theta=d \theta_{1} \cdots d \theta_{n},
$$

where $Z_{n}=\int_{\mathbb{T}^{n}} d \theta e^{-\sum_{j \neq k} f\left(\theta_{k}-\theta_{j}\right)}$. Denote the associated expectation functionals by $\mathbb{E}_{n}[-]$. By changing variables $z_{j}=\rho e^{i \theta_{j}}$ in Eq. (8) and $z_{j}=e^{i \theta_{j}}$ in Eq. (14), we get

$$
\mathcal{Z}_{n}(\vec{u} ; \vec{p})=a_{n} \mathbb{E}_{n}\left[\prod_{j=1}^{n} g\left(\rho, \theta_{j} ; \vec{u} ; \vec{p}\right)\right]
$$

where

$$
g(\rho, \theta ; \vec{u} ; \vec{p})=\prod_{m=1}^{s}\left(\rho e^{i \theta}-p_{m}\right) \prod_{\alpha=1}^{r} \frac{-u_{\alpha} \rho e^{i \theta}}{\left(\rho e^{i \theta}-u_{\alpha}\right)\left(q_{1} q_{2} \rho e^{i \theta}-u_{\alpha}\right)} .
$$

We estimate $\left|\mathcal{Z}_{n}(\vec{u} ; \vec{p})\right|$ by taking the absolute value inside. We arrive at

$$
\left|\mathcal{Z}_{n}(\vec{u} ; \vec{p})\right|^{\frac{1}{n}} \leq\left|a_{n}\right|^{\frac{1}{n}} \exp \left(\frac{1}{n} \log \mathbb{E}_{n}\left[e^{\sum_{j} \log \left|g\left(\rho, \theta_{j} ; \vec{u} ; p\right)\right|}\right]\right) .
$$

We estimate both factors on the right hand side separately. The first one is related to a known power series: 
Lemma 1. We have

$$
\limsup _{n \rightarrow \infty}\left|a_{n}\right|^{\frac{1}{n}}=1
$$

Proof. From [4] we know that

$$
\sum_{n \geq 0} a_{n} z^{n}=\exp \left(\sum_{n \geq 1} \frac{1-q_{1}^{n} q_{2}^{n}}{\left(1-q_{1}^{n}\right)\left(1-q_{2}^{n}\right)} \frac{z^{n}}{n}\right),
$$

as a formal power series. Since all coefficients are positive in this expansion and $q_{i}^{n} \rightarrow 0$ as $n \rightarrow \infty$ we see that the radius of convergence equals 1 .

These estimates already give a non-quantitative convergence result.

Lemma 2. Assume that $\lambda>0, q_{1}, q_{2}$ in the unit disk, both real or complex conjugate to each other. Then on each compact subset of the domain

$$
\left\{(\vec{u}, \vec{p}) \in \mathbb{C}^{r+s}|| q_{i}\left|\max _{\alpha}\right| u_{\alpha}\left|<\min _{\alpha}\right| u_{\alpha} \mid, \forall i=1,2\right\},
$$

the power series $\sum_{n=0}^{\infty} \mathcal{Z}_{n}(\vec{u} ; \vec{p}) z^{n}$ converges uniformly with a positive radius of convergence.

To prove this lemma notice that on such compact subsets $|g(\rho, \theta ; \vec{u} ; \vec{p})|$ is uniformly bounded on the integration circle $C_{\rho}$ for proper choice of $\rho$. Thus the coefficients $\mathcal{Z}_{n}(\vec{u} ; \vec{p})$ are bounded by const ${ }^{n}$.

To get a quantitative estimate of the radius of convergence we need to work harder. We have the following result:

Theorem 3. Let $h$ be a continuous, real-valued function on the torus $\mathbb{T}$. We have

$$
\frac{1}{n} \log \mathbb{E}_{n}\left[e^{\sum_{j} h\left(\theta_{j}\right)}\right] \rightarrow \frac{1}{2 \pi} \int_{\mathbb{T}} h(\theta) d \theta \quad(n \rightarrow \infty) .
$$

The proof of this theorem uses ideas from potential theory and is postponed to Sect. 2.7. It applies to a more general class of functions $f$ defining the probability measure, as we discuss in Sect. 3.2. Now we can apply Lemma 1 and Theorem 3 in Eq. (16) to estimate

$$
\limsup _{n \rightarrow \infty}\left|\mathcal{Z}_{n}(\vec{u} ; \vec{p})\right|^{\frac{1}{n}} \leq \exp \left(\frac{1}{2 \pi} \int_{\mathbb{T}} \log |g(\rho, \theta ; \vec{u} ; \vec{p})| d \theta\right) .
$$

Using that condition (7) says $\rho^{-1}\left|u_{\alpha}\right|<1$, but $\rho^{-1}\left|q_{1} q_{2}\right|^{-1}\left|u_{\alpha}\right|>1$, formula 18 from below implies

$$
\frac{1}{2 \pi} \int_{\mathbb{T}} \log |g(\rho, \theta ; \vec{u} ; p)| d \theta=\sum_{m=1}^{s} \max \left\{\log \left|p_{m}\right|, \log \rho\right\} .
$$

We obtain

$$
\limsup _{n \rightarrow \infty}\left|\mathcal{Z}_{n}(\vec{u} ; \vec{p})\right|^{\frac{1}{n}} \leq \prod_{m=1}^{s} \max \left\{\left|p_{m}\right|, \rho\right\}
$$

According to condition (7), the lower bound for $\rho$ is given by $\max _{\alpha}\left\{\left|u_{\alpha}\right|\right\}$. We let $\rho$ tend to this bound, completing the proof of Theorem 2 . 
2.7. Potential theory. In this section we prove Theorem 3 using techniques adapted from [13].

2.7.1. Setup of potential theory. Let $M(\mathbb{T})$ be the set of all Borel probability measures on $\mathbb{T}$ and $M_{0}(\mathbb{T})$ be the subset of all such measures which in addition do not contain point masses.

The function $f: \mathbb{T} \rightarrow \mathbb{R} \cup\{+\infty\}$ defined in Eq. (15) is continuous, bounded from below and has a single pole at $\theta=0$. Set

$$
I[\mu]=\iint_{\theta \neq \phi} f(\theta-\phi) d \mu(\theta) d \mu(\phi),
$$

where $\mu \in M(\mathbb{T})$. Since $f$ is bounded from below, $I$ is bounded from below, too. Define

$$
I_{0}:=\inf _{\mu \in M_{0}(\mathbb{T})} I[\mu]>-\infty .
$$

The aim of this section is to prove

Theorem 4. The normalized Lebesgue measure is the unique measure $\mu$ with $I[\mu]=I_{0}$. Moreover $I_{0}=0$.

Define the function $g_{\sigma}(\theta):=\frac{1}{2} \log \left(1+\sigma^{2}-2 \sigma \cos \theta\right)=\log \left|e^{i \theta}-\sigma\right|$ for $\sigma>0$. Its Fourier coefficients

$$
c_{k}\left(g_{\sigma}\right)=\frac{1}{2 \pi} \int_{\mathbb{T}} g_{\sigma}(\theta) e^{-i k \theta} d \theta= \begin{cases}-\frac{1}{2|k|} \min \left\{\sigma^{|k|}, \sigma^{-|k|}\right\}, & \text { if } k \neq 0, \\ \max \{0, \log \sigma\}, & \text { otherwise }\end{cases}
$$

are known. For $\sigma \neq 1$ see for example [7]. For the case $\sigma=1$, note that $g_{\sigma}$ is uniformly bounded from above and

$$
g_{\sigma}(\theta)=\log \left(1-2 \sigma+\sigma^{2}+2 \sigma-2 \sigma \cos (\theta)\right) \geq \log \sigma+g_{1}(\theta) .
$$

By dominated convergence, it suffices to show that $g_{1}$ is integrable. The only pole is at $\theta=0$. By changing variables to $x=2-2 \cos \theta$ we have to $\operatorname{consider} \log x \frac{1}{\sqrt{x}}$ which is integrable as one can see from integration by parts.

We write $q_{j}=\left|q_{j}\right| e^{i \tau_{j}}$. We have

$$
c_{k}(f)=-c_{k}\left(g_{1}\right)-c_{k}\left(g_{\left|q_{1} q_{2}\right|}\right)+e^{-i k \tau_{1}} c_{k}\left(g_{\left|q_{1}\right|}\right)+e^{-i k \tau_{2}} c_{k}\left(g_{\left|q_{2}\right|}\right) .
$$

Since $\left|q_{i}\right|<1$, we obtain $c_{0}(f)=0$, so in particular

Lemma 3. I vanishes for the normalized Lebesgue measure.

For $k \neq 0$ we get

$$
c_{k}(f)=\frac{1}{2|k|}\left(1+\left|q_{1} q_{2}\right|^{k}-e^{-i k \tau_{1}}\left|q_{1}\right|^{|k|}-e^{-i k \tau_{2}}\left|q_{2}\right|^{|k|}\right) .
$$

If $q_{1}=\overline{q_{2}}$, this is bounded from below by $\frac{1}{2|k|}\left(1-\left|q_{1} q_{2}\right|^{|k| / 2}\right)^{2}>0$. If $q_{1}, q_{2} \in(0,1)$ it equals $\frac{1}{2|k|}\left(1-\left|q_{1}\right|^{|k|}\right)\left(1-\left|q_{2}\right|^{|k|}\right)>0$. In either case we obtain 
Lemma 4. The Fourier coefficients $c_{k}(f)$ of $f \in L^{1}(\mathbb{T})$ satisfy $c_{k}(f)>0$ if $k \neq 0$ and $c_{0}(f)=0$. The Fourier series of $f$ converges everywhere except at $\theta=0$.

We use this to prove

Lemma 5. We have $I_{0}=0$.

Proof. Since the normalized Lebesgue measure is in $M_{0}(\mathbb{T})$ we have $I_{0} \leq 0$. On the other hand, for any $\mu \in M_{0}(\mathbb{T})$, we have

$$
\begin{aligned}
I[\mu] & =\iint f(\theta-\phi) d \mu(\theta) d \mu(\phi) \\
& =\sum_{k \neq 0} c_{k}(f) \iint e^{i(\theta-\phi)} d \mu(\theta) d \mu(\phi) \\
& =\sum_{k \neq 0} c_{k}(f)\left|c_{k}(\mu)\right|^{2} \geq 0 .
\end{aligned}
$$

Firstly, we have dropped the condition $\theta \neq \phi$ using the fact that $\mu$ does not contain point masses. Then we have applied Tonelli's theorem using the fact that $c_{k}(f) e^{i(\theta-\phi)}$ is bounded from below.

Lemma 6. The normalized Lebesgue measure is the unique measure $\mu \in M_{0}(\mathbb{T})$ for which $I_{0}=I[\mu]$.

Proof. Let $\mu, v \in M_{0}(\mathbb{T})$ with $I[\mu]=I[v]=I_{0}$. Since $I[\mu]$ and $I[v]$ are both finite,

$$
\begin{aligned}
I[\mu-\nu]= & \iint f(\theta-\phi) d \mu(\theta) d \mu(\phi)+\iint f(\theta-\phi) d v(\theta) d \nu(\phi) \\
& -\iint f(\theta-\phi) d \mu(\theta) d v(\phi)-\iint f(\theta-\phi) d v(\theta) d \mu(\phi) \in[-\infty, \infty)
\end{aligned}
$$

is well-defined. Using Tonelli's theorem for each summand, we get

$$
I[\mu-v]=\sum_{k \neq 0} c_{k}(f)\left|c_{k}(\mu)-c_{k}(v)\right|^{2} \geq 0 .
$$

In particular, $I[\mu-v]$ and all four terms in its expansion (19) are finite.

For $t \in[0,1]$ we have $v+t(\mu-v) \in M_{0}(\mathbb{T})$ and hence

$$
\begin{aligned}
0= & I_{0} \leq I[v+t(\mu-v)] \\
= & I[v]+t\left(\iint f(\theta-\phi) d v(\theta) d(\mu-v)(\phi)\right. \\
& \left.+\iint f(\theta-\phi) d(\mu-v)(\theta) d v(\phi)\right)+t^{2} I[\mu-v],
\end{aligned}
$$

where all terms are finite. The right hand side is a polynomial in $t$ which is nonnegative for $t \in[0,1]$ and vanishes at $t=0$ and $t=1$. We obtain $I[\mu-v] \leq 0$. From Eq. (20) we get $c_{k}(\mu)=c_{k}(v)$ for all $k \neq 0$, since $c_{k}(f)>0$ for $k \neq 0$. We obtain $\mu=v$ since $c_{0}(\mu)=c_{0}(\nu)$ trivially because $\mu$ and $\nu$ are probability measures.

The proof of Theorem 4 is complete. 
2.7.2. Application of potential theory. To a point $\theta \in \mathbb{T}^{n}$ we associate the probability measure

$$
\delta_{\theta}=\frac{1}{n} \sum_{j=1}^{n} \delta_{\theta_{j}}
$$

where on the right hand side we have a convex combination of ordinary Dirac measures. thus

Set $\mathbb{T}_{0}^{n}=\left\{\theta \in \mathbb{T}^{n}: \theta_{j} \neq \theta_{k}(j \neq k)\right\}$. For $\theta \in \mathbb{T}_{0}^{n}$ we have $\theta_{j} \neq \theta_{k} \Leftrightarrow j \neq k$ and

$$
n^{2} I\left[\delta_{\theta}\right]=\sum_{j \neq k} f\left(\theta_{j}-\theta_{k}\right)
$$

The intuition behind Theorem 3 is the following. The limit behavior for large $n$ of the quantity

$$
\frac{1}{n} \log \mathbb{E}_{n}\left[e^{\sum_{j} h\left(\theta_{j}\right)}\right]=\frac{1}{n} \log \frac{1}{Z_{n}} \int_{\mathbb{T}^{n}} d \theta e^{\sum_{j} h\left(\theta_{j}\right)} e^{-n^{2} I\left[\delta_{\theta}\right]}
$$

will be dominated by such $\theta \in \mathbb{T}^{n}$, for which $I\left[\delta_{\theta}\right]$ is close to $I_{0}=0$. This is the content of Lemma 7. Those $\delta_{\theta}$ will then for large $n$ equidistribute to approximate the normalized Lebesgue measure yielding $\frac{1}{2 \pi} \int_{\mathbb{T}} h(\theta) d \theta$. This will be the content of Lemma 8 and the discussion afterwards.

We use the notion of weak convergence for measures: We say a sequence of measures $\left(\mu_{n}\right)$ in $M(\mathbb{T})$ converges to a measure $\mu \in M(\mathbb{T})$ iff for all continuous and bounded functions $g$ on $\mathbb{T}$ we have

$$
\int_{\mathbb{T}} g d \mu_{n} \rightarrow \int_{\mathbb{T}} g d \mu .
$$

It is well-known that the space $M(\mathbb{T})$ with this notion of convergence is sequentially compact.

For $\eta>0$ define

$$
A_{n, \eta}=\left\{\theta \in \mathbb{T}_{0}^{n}: I_{0} \leq I\left[\delta_{\theta}\right] \leq I_{0}+\eta\right\}=\left\{\theta \in \mathbb{T}^{n}: \sum_{j \neq k} f\left(\theta_{k}-\theta_{j}\right) \leq \eta n^{2}\right\} .
$$

This set is compact. Denote by $P_{n}[S]$ the measure of a measurable set $S \subset \mathbb{T}^{n}$ with respect to the probability measure $P_{n}$. We have

Lemma 7. $0 \leq P_{n}\left[\mathbb{T}^{n} \backslash A_{n, \eta}\right] \leq e^{-\eta n^{2}}$.

Proof. We have

$$
\int_{\mathbb{T}^{n} \backslash A_{n, \eta}} d \theta e^{-\sum_{j \neq k} f\left(\theta_{k}-\theta_{j}\right)} \leq \int_{\mathbb{T}^{n} \backslash A_{n, \eta}} d \theta e^{-\eta n^{2}} \leq(2 \pi)^{n} e^{-\eta n^{2}} .
$$

Let $\mu$ denote the normalized Lebesgue measure. By the Jensen inequality we have

$$
\frac{Z_{n}}{(2 \pi)^{n}}=\int_{\mathbb{T}_{0}^{n}} \prod_{j} d \mu\left(\theta_{j}\right) e^{-\sum_{j \neq k} f\left(\theta_{k}-\theta_{j}\right)}
$$




$$
\geq \exp \left(\int_{\mathbb{T}_{0}^{n}} \prod_{j} d \mu\left(\theta_{j}\right)\left(-\sum_{j \neq k} f\left(\theta_{k}-\theta_{j}\right)\right)\right)
$$

Now

$$
\begin{aligned}
-\sum_{j \neq k} \int_{\mathbb{T}_{0}^{n}} \prod_{j^{\prime}} d \mu\left(\theta_{j^{\prime}}\right) f\left(\theta_{k}-\theta_{j}\right) & =-n(n-1) \iint f(\theta-\phi) d \mu(\theta) d \mu(\phi) \\
& =-n(n-1) I[\mu]=0 .
\end{aligned}
$$

Moreover, we have

Lemma 8. If the measures $v_{n, \eta}$ are Dirac measures supported at $\tau^{n, \eta} \in A_{n, \eta}$ and $v_{n_{k}, \eta} \rightarrow v_{\eta}$ is a convergent subsequence, we have $v_{\eta} \in M_{0}(\mathbb{T})$ and $I\left[v_{\eta}\right] \leq \eta$. If $v_{\eta_{k}} \rightarrow v$ is a convergent sequence with $\eta_{k} \rightarrow 0$, the limit $v$ has to be the normalized Lebesgue measure.

Proof. Let $L \in \mathbb{R}$ and separate the diagonal part:

$$
\begin{aligned}
\eta \geq I\left[v_{n, \eta}\right] & =\iint_{\theta \neq \phi} f(\theta-\phi) d v_{n, \eta}(\theta) d v_{n, \eta}(\phi) \\
& \geq \iint_{\theta \neq \phi} \min \{f(\theta-\phi), L\} d v_{n, \eta}(\theta) d v_{n, \eta}(\phi) \\
& =\frac{1}{n^{2}} \sum_{j \neq k} \min \left\{f\left(\tau_{j}^{n, \eta}-\tau_{k}^{n, \eta}\right), L\right\} \\
& =\iint \min \{f(\theta-\phi), L\} d v_{n, \eta}(\theta) d v_{n, \eta}(\phi)-\frac{L}{n} .
\end{aligned}
$$

Let $\epsilon>0$. Using the Weierstrass approximation theorem, pick a polynomial $p(\theta, \phi)$ which uniformly approximates the last integrand up to an error of $\epsilon$. For $n=n_{k}$ we get

$$
\eta \geq \iint p(\theta, \phi) d v_{n_{k}, \eta}(\theta) d v_{n_{k}, \eta}(\phi)-\epsilon-\frac{L}{n_{k}} .
$$

Send $k \rightarrow \infty$ to get

$$
\eta \geq \iint p(\theta, \phi) d v_{\eta}(\theta) d v_{\eta}(\phi)-\epsilon \geq \iint \min \{f(\theta-\phi), L\} d v_{\eta}(\theta) d v_{\eta}(\phi)-2 \varepsilon .
$$

Now send $\epsilon \rightarrow 0$ and let $L \rightarrow \infty$. By monotonicity the limit can pass to the integrand. We obtain $v_{\eta} \in M_{0}(\mathbb{T})$ and

$$
\eta \geq \iint f(\theta-\phi) d v_{\eta}(\theta) d v_{\eta}(\phi)=\iint_{\phi \neq \theta} f(\theta-\phi) d v_{\eta}(\theta) d v_{\eta}(\phi)=I\left[v_{\eta}\right] .
$$

Now let $v_{\eta_{k}} \rightarrow v$ be a convergent sequence with $\eta_{k} \rightarrow 0$. Again fix $L \in \mathbb{R}$ and estimate, using $v_{\eta_{k}} \in M_{0}(\mathbb{T})$,

$$
\eta_{k} \geq I\left[v_{\eta_{k}}\right]=\iint f(\theta-\phi) d v_{\eta}(\theta) d v_{\eta}(\phi) \geq \iint \min \{L, f(\theta-\phi)\} d v_{\eta}(\theta) d v_{\eta}(\phi) .
$$


Let $\epsilon>0$. Using Weierstrass we get in the limit $k \rightarrow \infty$

$$
0 \geq \iint \min \{L, f(\theta-\phi)\} d v(\theta) d v(\phi)-2 \epsilon .
$$

Again, we let $\epsilon \rightarrow 0$ and $L \rightarrow \infty$ to get $\nu \in M_{0}(\mathbb{T})$ and $0 \geq I[v]$. The claim follows.

Now we can prove Theorem 3. Let $h$ be a continuous function on the torus. Fix $\eta>0$. By Lemma 7, we have

$$
\limsup _{n \rightarrow \infty} \frac{1}{n} \log \mathbb{E}_{n}\left[e^{\sum_{j} h\left(\theta_{j}\right)}\right]=\limsup _{n \rightarrow \infty} \frac{1}{n} \log \int_{A_{n, \eta}} d \theta P_{n}(\theta) e^{\sum_{j} h\left(\theta_{j}\right)},
$$

and analogously for lim inf. Let the continuous, real-valued function $e^{\sum_{j} h\left(\theta_{j}\right)}$ on the compact set $A_{n, \eta}$ attain its maximum at $\tau^{n, \eta} \in A_{n, \eta}$ and its minimum at $\sigma^{n, \eta} \in A_{n, \eta}$. Denote Dirac measures by $\nu_{n, \eta}$ and $\lambda_{n, \eta}$. We have

$$
\frac{1}{n} \log \int_{A_{n, \eta}} d \theta P_{n}(\theta) e^{\sum_{j} h\left(\theta_{j}\right)} \leq \frac{1}{n} \log P_{n}\left[A_{n, \eta}\right] e^{\sum_{j} h\left(\tau_{j}^{n, \eta}\right)} \leq \int_{\mathbb{T}} d v_{n, \eta}(\theta) h(\theta) .
$$

Similarly,

$$
\frac{1}{n} \log \int_{A_{n, \eta}} d \theta P_{n}(\theta) e^{\sum_{j} h\left(\theta_{j}\right)} \geq \frac{1}{n} \log P_{n}\left[A_{n, \eta}\right] e^{\sum_{j} h\left(\sigma_{j}^{n, \eta}\right)} \geq \int_{\mathbb{T}} d \lambda_{n, \eta}(\theta) h(\theta)+O\left(\frac{1}{n}\right)
$$

since by Lemma $7, P_{n}\left[A_{n, \eta}\right] \geq 1-e^{-\eta n^{2}} \rightarrow 1$. Let $n_{k}$ define a subsequence with

$$
\limsup _{n \rightarrow \infty} \frac{1}{n} \log \int_{A_{n, \eta}} d \theta P_{n}(\theta) e^{\sum_{j} h\left(\theta_{j}\right)}=\lim _{k \rightarrow \infty} \frac{1}{n_{k}} \log \int_{A_{n_{k}, \eta}} d \theta P_{n_{k}}(\theta) e^{\sum_{j} h\left(\theta_{j}\right)} .
$$

Let $m_{k}$ define a subsequence that realizes the corresponding lim inf. By passing to respective subsequences, we can suppose that $\nu_{n_{k}, \eta} \rightarrow \nu_{\eta}$ and $\lambda_{m_{k}, \eta} \rightarrow \lambda_{\eta}$ for some Borel probability measures $\lambda_{\eta}, v_{\eta}$. We obtain

$$
\begin{aligned}
\int_{\mathbb{T}} h(\theta) d \lambda_{\eta}(\theta) & \leq \liminf _{n \rightarrow \infty} \frac{1}{n} \log \mathbb{E}_{n}\left[e^{\sum_{j} h\left(\theta_{j}\right)}\right] \\
& \leq \limsup _{n \rightarrow \infty} \frac{1}{n} \log \mathbb{E}_{n}\left[e^{\sum_{j} h\left(\theta_{j}\right)}\right] \leq \int_{\mathbb{T}} h(\theta) d v_{\eta}(\theta) .
\end{aligned}
$$

The parameter $\eta>0$ is arbitrary. Now let $\eta_{k} \rightarrow 0$ define a subsequence $v_{\eta} \rightarrow v$ and $\eta_{k}^{\prime} \rightarrow 0$ define a subsequence $\lambda_{\eta} \rightarrow \lambda$. By Lemma $8, v=\lambda$ is the normalized Lebesgue measure. We obtain

$$
\begin{aligned}
\frac{1}{2 \pi} \int_{\mathbb{T}} h(\theta) d \theta & \leq \liminf _{n \rightarrow \infty} \frac{1}{n} \log \mathbb{E}_{n}\left[e^{\sum_{j} h\left(\theta_{j}\right)}\right] \\
& \leq \limsup _{n \rightarrow \infty} \frac{1}{n} \log \mathbb{E}_{n}\left[e^{\sum_{j} h\left(\theta_{j}\right)}\right] \leq \frac{1}{2 \pi} \int_{0}^{2 \pi} h(\theta) d \theta .
\end{aligned}
$$

The proof of Theorem 3 is complete. 
Remark 2. A physical interpretation of Theorem 3 goes as follows: The points of $\mathbb{T}^{n}$ are the coordinates of $n$ particles on the torus $\mathbb{T}$ that interact via the two body potential $f\left(\theta_{j}-\theta_{k}\right)$. The integral over $\mathbb{T}^{n}$ on the left hand side of Eq. (17) is dominated by particle configurations which minimize the potential energy of the system. The even function $f(\theta)$ has a pole at $\theta=0$ and one minimum $\theta_{0}$ in $(0, \pi)$. Hence, the particles experience a strong repulsive force once they get close to each other and have a preferred distance $\theta_{0}$ from each other. For large $n$ they cannot all stay in their preferred distance since the torus is compact. Hence, the repulsive part dominates and in the limit of large $n$ the particles equidistribute. The evaluation of the left hand side of Eq. (17) on those equidistributed points on $\mathbb{T}$ defines a Riemann sum approximating the integral on the right hand side of Eq. (17).

2.8. Identification of coefficients and conclusion. We evaluate the formal parameters of Sect. 2.5 at the complex parameters of Sect. 2.6.1. We set

$$
t_{i}^{-1}=e^{-\lambda \epsilon_{i}}=q_{i}, \quad e_{\alpha}^{-1}=e^{-\lambda a_{\alpha}}=u_{\alpha}, \quad b_{m}=e^{\lambda w_{m}}=p_{m} .
$$

Under the assumption $\lambda>0$, the conditions stated at the beginning of Sect. 2.6.1 are satisfied provided we assume $\operatorname{Re} \epsilon_{i}>0$ and $\left(\epsilon_{1}=\overline{\epsilon_{2}}\right.$ or $\left.\epsilon_{1}, \epsilon_{2} \in \mathbb{R}\right)$ and

$$
\max _{\alpha} \operatorname{Re}\left(a_{\alpha}\right)-\min _{\alpha} \operatorname{Re}\left(a_{\alpha}\right)<\operatorname{Re} \epsilon_{i}, \quad i=1,2 .
$$

Hence, $\mathcal{Z}_{n}(\vec{u} ; \vec{p})$ is well-defined. To ensure that each simple residue $\mathcal{Z}_{\vec{Y}}(\vec{u} ; \vec{p})$ is welldefined, we have to require conditions (11), which translate to

$$
\begin{array}{lr}
a_{\alpha}-a_{\beta} \not \equiv x \epsilon_{1}+y \epsilon_{2}, & \forall \alpha \neq \beta \in\{1, \ldots, r\} \quad \forall x, y \in\{-n, \ldots, n\}, \\
x \epsilon_{1} \not \equiv(y+1) \epsilon_{2},(x+1) \epsilon_{1} \not \equiv y \epsilon_{2}, & \forall x, y \in\{0, \ldots, n-1\},
\end{array}
$$

where the inequalities are modulo $\frac{2 \pi i}{\lambda} \mathbb{Z}$. Under these conditions, Theorem 1 implies $Z_{n}\left(\epsilon_{1}, \epsilon_{2}, \vec{a} ; \vec{w} ; \lambda\right)=\mathcal{Z}_{n}(\vec{u} ; \vec{p})$. By Remark 1 , the sum $Z_{n}\left(\epsilon_{1}, \epsilon_{2}, \vec{a} ; \vec{w}\right)$ is well-defined even if we drop condition (21). The representation of the coefficients as integrals allowed us to apply the potential theory in Sect. 2.6.3 to estimate their growth in Theorem 2. We obtain

Theorem 5. Let $\lambda>0$ and $\epsilon_{1}$ and $\epsilon_{2}$ be a pair of complex numbers with positive real part. Assume either they are complex conjugate or both real. The K-theoretic Nekrasov partition function $Z\left(\epsilon_{1}, \epsilon_{2}, \vec{a} ; \vec{w}, \mathfrak{q}, \lambda\right)$ given by Eq. (3) is an analytic function of $\mathfrak{q}, \vec{a}, \vec{w}$ in the domain

$$
\begin{array}{r}
\vec{w} \in \mathbb{C}^{s}, \quad \max _{\alpha} \operatorname{Re}\left(a_{\alpha}\right)-\min _{\alpha} \operatorname{Re}\left(a_{\alpha}\right)<\operatorname{Re} \epsilon_{i}, \quad i=1,2, \\
|\mathfrak{q}|<\lambda^{s-2 r} e^{\lambda\left(r \frac{\epsilon_{1}+\epsilon_{2}}{2}+\sum_{m=1}^{s} \min \left\{-\operatorname{Re} w_{m}, \operatorname{Re} a_{1}, \ldots, \operatorname{Re} a_{r}\right\}\right)} .
\end{array}
$$

There remains to prove the analyticity in $\vec{a}, \vec{w}$. By Lemma 2 , we know that in a neighbourhood $U$ of each $(\vec{a}, \vec{w})$ the series defining the partition functions converges to an analytic function for $|\mathfrak{q}|<r$ for some small positive radius $r=r(U)$. On the other hand, Theorem 2 tells us that we have convergence of the power series for the indicated range of $\mathfrak{q}$ and fixed $\vec{a}, \vec{w}$ in the domain. By Hartogs' lemma ([6], Theorem 2, p. 139) the series converges to an analytic function on the whole domain. 


\section{Generalization}

Our technique can be generalized to different parameter ranges and similar types of gauge theory.

\subsection{Different parameter ranges. Assume $\vec{p}=\emptyset$. Under the inversion}

$$
\left(q_{1}, q_{2}, u_{1}, \ldots, u_{r}\right) \mapsto\left(q_{1}^{-1}, q_{2}^{-1}, u_{1}^{-1}, \ldots, u_{r}^{-1}\right)
$$

the coefficient $\mathcal{Z}_{n}(\vec{u} ; \vec{p}=\emptyset)$ gets multiplied by $\left(q_{1} q_{2}\right)^{-n r}$. This can directly be seen from Eq. (13). Using this observation, bounds for $\left|q_{1}\right|,\left|q_{2}\right|>1$ can be obtained as well.

3.2. Similar types of gauge theory. Our technique readily generalizes to partition functions of gauge theories where the weight factor in line (9) in coordinates $z_{j}=\rho e^{i \theta_{j}}$ is of the form

$$
\exp \left(-\sum_{j \neq k} f\left(\theta_{j}-\theta_{k}\right)\right),
$$

where $f: \mathbb{T} \rightarrow \mathbb{R} \cup\{+\infty\}$ is continuous, bounded from below, has a single pole at $\theta=0$ and its Fourier coefficients $c_{k}(f)$ satisfy $c_{k}(f)>0$ for $k \neq 0$ and $c_{0}(f)=0$.

An example would be $\mathcal{N}=2^{*}$ supersymmetric gauge theory, i.e. with massive matter in the adjoint representation [18, equation (3.25)]. One has to consider a different polynomial in the numerator of the integrand in (8) and multiply the weight in line (9) by

$$
\prod_{1 \leq j<k \leq n} \frac{\left(z_{j}-q_{1} m^{-1} z_{k}\right)\left(z_{j}-q_{2} m^{-1} z_{k}\right)\left(z_{j}-q_{1}^{-1} m z_{k}\right)\left(z_{j}-q_{2}^{-1} m z_{k}\right)}{\left(z_{j}-m z_{k}\right)\left(z_{j}-m^{-1} z_{k}\right)\left(z_{j}-q_{1} q_{2} m^{-1} z_{k}\right)\left(z_{j}-q_{1}^{-1} q_{2}^{-1} m z_{k}\right)} .
$$

\section{Specialization: Norm of Deformed Gaiotto States}

In this section, we briefly discuss an application of our results to conformal field theory, namely the finiteness of the norm of deformed Gaiotto states.

4.1. Definition. In this introduction, let $q, t$ denote two generic complex parameters. We follow the exposition of [3]. The deformed Virasoro algebra $V i r_{q, t}$ is defined as the associative algebra topologically generated by $T_{n}, n \in \mathbb{Z}$ with the defining relations

$$
\begin{aligned}
{\left[T_{n}, T_{m}\right]=} & -\sum_{l=1}^{\infty} r_{l}\left(T_{n-l} T_{m+l}-T_{m-l} T_{n+l}\right) \\
& -\frac{(1-q)\left(1-t^{-1}\right)}{1-q t^{-1}}\left(q^{n} t^{-n}-q^{-n} t^{n}\right) \delta_{m+n, 0} .
\end{aligned}
$$

The coefficients $r_{l}$ are determined by the expansion

$$
r(x)=\sum_{l \geq 0} r_{l} x^{l}=\exp \left(\sum_{n \geq 1} \frac{\left(1-q^{n}\right)\left(1-t^{-n}\right)}{1+q^{n} t^{-n}} \frac{x^{n}}{n}\right) .
$$


For $h \in \mathbb{C}$, the Verma module $M_{h}$ is generated by a vector $|h\rangle$ with $T_{0}|h\rangle=h|h\rangle$ and $T_{n}|h\rangle=0$ if $n \geq 1$. The operator $T_{0}$ defines a grading $M_{h}=\bigoplus_{n \geq 0} M_{h, n}$ where $M_{h, n}$ is the eigenspace of $T_{0}$ corresponding to the eigenvalue $h+n$. As usual, each $M_{h, n}$ has a basis

$$
T_{\lambda}|h\rangle:=T_{-\lambda(1)} \cdots T_{-\lambda(l)}|h\rangle
$$

indexed by partitions $\lambda=(\lambda(1), \ldots, \lambda(l))$ of size $|\lambda|=n$. The Shapovalov form $S: M_{h} \otimes M_{h} \rightarrow \mathbb{C}$ is characterized by $S(|h\rangle,|h\rangle)=1$ and $S\left(T_{n} x, y\right)=S\left(x, T_{-n} y\right)$ for $x, y \in M_{h}$ and $n \in \mathbb{Z}$. The decomposition $M_{h}=\bigoplus_{n \geq 0} M_{h, n}$ is orthogonal with respect to the Shapovalov form. In particular, the so-called Kac matrix with entries $S_{\lambda, \mu}:=S\left(T_{\lambda}|h\rangle, T_{\mu}|h\rangle\right)$ is block diagonal with finite blocks $\left(S_{\lambda, \mu}^{(n)}\right)_{|\lambda|,|\mu|=1, \ldots, n}$.

A deformed Gaiotto state is a formal power series $|G\rangle=\sum_{n \geq 0} \xi^{n}\left|G_{n}\right\rangle$ whose coefficients $\left|G_{n}\right\rangle \in M_{h, n}$ satisfy $T_{1}\left|G_{n}\right\rangle=\left|G_{n-1}\right\rangle$ with $\left|G_{-1}\right\rangle:=0$ and $T_{n}\left|G_{m}\right\rangle=0$ for $n \geq 2$ and all $m$. In terms of the expansion $\left|G_{n}\right\rangle=\sum_{|\lambda|=n} g_{\lambda}^{(n)} T_{\lambda}|h\rangle$ these conditions read

$$
\sum_{|\lambda|=n} g_{\lambda}^{(n)} S_{\lambda, \mu}^{(n)}=\delta_{\mu\left(1^{n}\right)}, \quad n=0,1, \ldots
$$

where $\left(1^{n}\right)$ is the partition $(1, \ldots, 1)$ of size $n$.

From now on, we assume $q, t \neq 1$. The zeros of the determinant of the $n$-th block $S^{(n)}$ of the Kac matrix are located at [23, equation (2.4)]

$$
h= \pm\left(t^{r / 2} q^{-s / 2}+t^{-r / 2} q^{s / 2}\right), \quad r, s \geq 1, r s=n
$$

Outside of these sets, the Kac matrix is invertible, hence the Gaiotto state exists uniquely and its norm with respect to the Shapovalov form is given by the formal power series

$$
S(|G\rangle,|G\rangle)=\sum_{n \geq 0} \xi^{2 n}\left(S^{(n)}\right)_{\left(1^{n}\right),\left(1^{n}\right)}^{-1} .
$$

Let $Q \in \mathbb{C}$ with $h=Q^{\frac{1}{2}}+Q^{-\frac{1}{2}}$. From the AGT relation [3,23,24] we obtain the formal expansion as a Nekrasov partition function

$$
S(|G\rangle,|G\rangle)=\sum_{n \geq 0} \xi^{2 n} q^{-n} t^{n} Z_{n}(q, t, Q),
$$

where the coefficients are sums over pairs $(\nu, \mu)$ of partitions:

$$
\begin{aligned}
Z_{n}(q, t, Q) & =\sum_{|v|+|\mu|=n} \frac{1}{N_{\nu, \mu}(Q) N_{\mu, \nu}\left(Q^{-1}\right) N_{\nu, v}(1) N_{\mu, \mu}(1)}, \\
N_{\nu, \mu}(Q) & =\prod_{t \in \mu}\left(1-Q q^{a_{v}(t)} t^{l_{\mu}(t)+1}\right) \prod_{s \in v}\left(1-Q q^{-a_{\mu}(s)-1} t^{-l_{\nu}(s)}\right) .
\end{aligned}
$$


4.2. Finiteness of norm. We want to recover the coefficients defined in Eq. (8). We set $q_{1}=t, q_{2}=q^{-1}, r=2$ and $u_{1}=u_{2}^{-1}=Q^{\frac{1}{2}}$. The conditions stated at the beginning of Sect. 2.6.1 say $|q|>1,|t|<1$ and $(t \bar{q}=1$ or $t, q>0)$ and

$$
|t| \max \left\{|Q|,|Q|^{-1}\right\}<\min \left\{|Q|,|Q|^{-1}\right\}, \quad \max \left\{|Q|,|Q|^{-1}\right\}<|q| \min \left\{|Q|,|Q|^{-1}\right\} .
$$

The condition (11) on the well-definedness of all simple residues translates to

$$
Q \notin\left\{q^{x} t^{y}: x, y \in \mathbb{Z}\right\}, \text { and } q^{x} \neq t^{y} \forall(x, y) \in \mathbb{Z}^{2} \backslash\{(0,0)\} .
$$

We extend the condition on $Q$ on the left to $-Q$, thus excluding all poles of the Kac determinant in Eq. (22). The condition on the right we drop by remark (1). From Eq. (12) in Theorem 1, we then recover

$$
S(|G\rangle,|G\rangle)=\sum_{n \geq 0} z^{n} \mathcal{Z}_{n}(\vec{u} ; p=\emptyset)
$$

for $z=\xi^{2} t q^{-1}$. Hence, Theorem 2 implies that series (24) converges for $|z|<1$. We obtain

Theorem 6. Let $q$ and $t$ be a pair of complex numbers of with $|t|<1$ and $|q|>1$ Suppose either $t \bar{q}=1$ or $t, q>0$. Let $Q^{\frac{1}{2}}$ be a complex number such that

$$
\begin{array}{ll}
Q \notin\left\{q^{x} t^{y}: x, y \in \mathbb{Z}\right\}, & |q| \max \left\{|Q|,|Q|^{-1}\right\}<\min \left\{|Q|,|Q|^{-1}\right\}, \\
-Q \notin\left\{q^{x} t^{y}: x, y \in \mathbb{Z}\right\}, & \max \left\{|Q|,|Q|^{-1}\right\}<|t| \min \left\{|Q|,|Q|^{-1}\right\} .
\end{array}
$$

Set $h=Q^{\frac{1}{2}}+Q^{-\frac{1}{2}}$. The deformed Gaiotto state $|G\rangle$ with formal parameter $\xi \in \mathbb{C}$ exists for the Verma module $M_{h}$ for the deformed Virasoro algebra Vir $r_{q, t}$, and its norm $S(|G\rangle,|G\rangle)$ is an analytic function in the variable $\xi$ for

$$
|\xi|<|t|^{-\frac{1}{2}}|q|^{\frac{1}{2}}
$$

\section{Open Problems}

Here, we briefly describe two formal power series, to which we intended to apply our results. Their coefficients are certain limits of the coefficients we studied earlier. Due to the lack of uniformity in our estimates, we were not able to prove convergence of those power series.

5.1. Homological version of Nekrasov partition function. The $K$-theoretic Nekrasov partition function defined in Sect. 2 has a counterpart in which $K$-theory groups are replaced by $\tilde{T}$-equivariant Borel-Moore homology groups $H_{*}^{\tilde{T}}(-)$. All $H_{*}^{\tilde{T}}(-)$ are modules for the ring

$$
S(\tilde{T}):=H_{*}^{\tilde{T}}(p t) \cong \mathbb{Z}\left[\epsilon_{1}, \epsilon_{2}, a_{1}, \ldots, a_{r}\right] .
$$

Let $\mathcal{S} \cong \mathbb{Q}\left(\epsilon_{1}, \epsilon_{2}, a_{1}, \ldots, a_{r}\right)$ denote its quotient field. The maps $\iota$ and $\iota_{\vec{Y}}$, defined in Sect. 2, all define pushforwards in equivariant homology. The localization theorem in equivariant homology says that $\iota$ becomes an isomorphism after tensoring with $\mathcal{S}$. 
The fixed point theorem says that after tensoring with $\mathcal{S}$ we have

$$
\left(\iota_{*}\right)^{-1}=\sum_{|\vec{Y}|=n} \frac{\iota_{\vec{Y}}^{*}}{e\left(T_{\vec{Y}} M(r, n)\right)},
$$

where $e(-)$ denotes the Euler class. The homological Nekrasov partition function for pure Yang Mills theory is defined as a formal power series

$$
Z\left(\epsilon_{1}, \epsilon_{2}, \vec{a} ; \mathfrak{q}\right)=\sum_{n \geq 0} \mathfrak{q}^{n} Z_{n}\left(\epsilon_{1}, \epsilon_{2}, \vec{a}\right)
$$

with coefficients $Z_{n}\left(\epsilon_{1}, \epsilon_{2}, \vec{a}\right)=\sum_{|\vec{Y}|=n}\left(\iota_{*}\right)^{-1}[M(r, n)]$ where $[M(r, n)]$ denotes the fundamental class of $M(r, n)$. Using the fixed point formula (25) and Eq. (2) to compute the Euler classes gives

$$
Z_{n}\left(\epsilon_{1}, \epsilon_{2}, \vec{a}\right)=\sum_{|\vec{Y}|=n} \frac{1}{\prod_{\alpha, \beta} n_{\alpha, \beta}^{\vec{Y}}\left(\epsilon_{1}, \epsilon_{2}, \vec{a}\right)},
$$

where

$$
\begin{aligned}
n_{\alpha, \beta}^{\vec{Y}}\left(\epsilon_{1}, \epsilon_{2}, \vec{a}\right)= & \prod_{s \in Y_{\alpha}}\left(-l_{Y_{\beta}}(s) \epsilon_{1}+\left(a_{Y_{\alpha}}(s)+1\right) \epsilon_{2}+a_{\alpha}-a_{\beta}\right) \\
& \prod_{t \in Y_{\beta}}\left(\left(l_{Y_{\alpha}}(t)+1\right) \epsilon_{1}-a_{Y_{\beta}}(t) \epsilon_{2}+a_{\alpha}-a_{\beta}\right) .
\end{aligned}
$$

In particular, the coefficients of the homological Nekrasov partition function are limits of the coefficients of the $K$-theoretic partition function, as defined in Eq. (5):

$$
\lambda^{2 r n} Z_{n}\left(\epsilon_{1}, \epsilon_{2}, \vec{a} ; \vec{w}=\emptyset ; \lambda\right) \rightarrow Z_{n}\left(\epsilon_{1}, \epsilon_{2}, \vec{a}\right) \quad(\lambda \rightarrow 0) .
$$

Equation (27) suggests that, under the same conditions on $\epsilon_{1}, \epsilon_{2}, a_{1}, \ldots, a_{r}$ as stated in Theorem 5, the homological Nekrasov partition function $Z\left(\epsilon_{1}, \epsilon_{2}, \vec{a} ; \mathfrak{q}\right)$ converges for all $\mathfrak{q} \in \mathbb{C}$. This question remains unanswered: Neither is the limit (27) uniform in $n$, nor is the estimate for the radius of convergence uniform in $\lambda$.

Remark 3. In the special case $\epsilon_{1}+\epsilon_{2}=0$, one can directly estimate the coefficients $Z_{n}$ to prove convergence of the expansion $Z\left(\epsilon_{1}, \epsilon_{2}, \vec{a} ; \mathfrak{q}\right)=\sum_{n \geq 0} \mathfrak{q}^{n} Z_{n}\left(\epsilon_{1}, \epsilon_{2}, \vec{a}\right)$ : The diagonal term $\alpha=\beta$ in $\prod_{\alpha, \beta=1}^{n} n_{\alpha, \beta}^{\vec{Y}}\left(\epsilon_{1}, \epsilon_{2}, \vec{a}\right)$ is a product over all hook lengths occurring in the Young diagram $Y_{\alpha}$. By the hook length formula we obtain $\frac{1}{\left|Y_{\alpha}\right| !}$ times the Plancherel measure of the Young diagram $Y_{\alpha}$, which allows one to estimate the expansion for all $\mathfrak{q} \in \mathbb{C}$, see [12, proposition 1]. This technique, which requires $\epsilon_{1}+\epsilon_{2}=0$, also generalizes [5] to the $K$-theoretic Nekrasov partition function without additional matter fields. 


\subsection{Conformal blocks.}

5.2.1. The Virasoro algebra and Verma modules. We consider the Virasoro algebra Vir. It is a complex Lie algebra with generators $L_{n}, n \in \mathbb{Z}$ and central element $C$ satisfying

$$
\left[L_{m}, L_{n}\right]=(m-n) L_{m+n}+\frac{1}{12}\left(m^{3}-m\right) \delta_{m+n, 0} C .
$$

Fix $c \in \mathbb{C}$, the so-called central charge. It will remain unchanged for the remainder of this introduction. For $h \in \mathbb{C}$ the Verma module $V_{h}$ for the Virasoro algebra of conformal dimension $h$ and central charge $c$ is a module generated by a vector $|h\rangle$ satisfying $L_{0}|h\rangle=h|h\rangle, C|h\rangle=c|h\rangle$ and $L_{n}|h\rangle=0$ for $n \geq 1$. A basis is given by $L_{\lambda}|h\rangle:=$ $L_{-\lambda(1)} \cdots L_{-\lambda(l)}|h\rangle$, where $\lambda=(\lambda(1), \ldots, \lambda(l))$ is a partition. The Verma module comes with a bilinear form, the Shapovalov form, $S: V_{h} \otimes V_{h} \rightarrow \mathbb{C}$ characterized by $S\left(L_{n} x, y\right)=S\left(x, L_{-n} y\right)$, for $x, y \in V_{h}$ and $S(|h\rangle,|h\rangle)=1$.

5.2.2. Intertwiners. Fix complex numbers $h, h_{1}, h_{2}$ and set $a=h_{2}-h_{1}-h$. Given two Verma modules $V_{h_{1}}$ and $V_{h_{2}}$ for the same central charge $c$, an intertwiner $\phi(z)=$ $\sum_{n \in \mathbb{Z}} \phi_{n} z^{n+a}$ between $V_{h_{1}}$ and $V_{h_{2}}$ of conformal dimension $h$ is a formal power series whose coefficients $\phi_{n}$ are linear maps $V_{h_{1}} \rightarrow V_{h_{2}}$ such that

$$
\left[L_{n}, \phi(z)\right]=\left(z^{n+1} \partial_{z}+(n+1) h z^{n}\right) \phi(z) .
$$

In conformal field theory, this property models transformations of fields under infinitesimal conformal transformations. Conformal blocks model vacuum expectation values of such fields. Mathematically they are defined as follows:

5.2.3. Conformal blocks. Let $h_{l}, H_{l}, h_{m}, H_{r}, h_{r}$ be complex numbers. Let $\phi_{l}(w)$ be an intertwiner of conformal dimension $H_{l}$ from $V_{h_{m}}$ to $V_{h_{l}}$. Let $\phi_{r}(z)$ be an intertwiner of conformal dimension $H_{r}$ from $V_{h_{r}}$ to $V_{h_{m}}$. The object

$$
\left\langle h_{l}\left|\phi_{l}(1) \phi_{r}(z)\right| h_{r}\right\rangle:=\lim _{w \rightarrow 1} S\left(\left|h_{l}\right\rangle, \phi_{l}(w)\left(\phi_{r}(z)\left|h_{r}\right\rangle\right)\right)
$$

is called a four-point conformal block. It defined as $z^{h-h_{r}-H_{r}}$ times a formal power series in $z$, whose complex coefficients are in principle determined by Eq. (28) up to normalization. The limit is taken for each coefficient separately.

The AGT relation allows us to express the conformal block as a Nekrasov partition function: One first has to introduce the Liouville parametrization of the conformal dimensions and the central charge. We pick $b \in \mathbb{C}$ with

$$
c=1+6\left(b+b^{-1}\right)^{2},
$$

to parametrize the central charge. The conformal dimensions $h_{l}, h_{m}, h_{r}$ of the Verma modules $V_{h_{l}}, V_{h_{m}}$ and $V_{h_{r}}$ are parametrized as

$$
h_{l}=\frac{\left(b+b^{-1}\right)^{2}}{4}-P_{l}^{2}, \quad h_{m}=\frac{\left(b+b^{-1}\right)^{2}}{4}-P_{m}^{2}, \quad h_{l}=\frac{\left(b+b^{-1}\right)^{2}}{4}-P_{l}^{2},
$$

where $P_{l}, P_{m}, P_{r} \in \mathbb{C}$. The conformal dimensions $H_{l}$ and $H_{r}$ of the intertwiners $\phi_{l}(w)$ and $\phi_{r}(z)$ are parametrized as

$$
H_{r}=\alpha_{r}\left(b+b^{-1}-\alpha_{r}\right), \quad H_{l}=\alpha_{l}\left(b+b^{-1}-\alpha_{l}\right),
$$


where $\alpha_{r}, \alpha_{l} \in \mathbb{C}$. We have [1]

$$
\left\langle h_{l}\left|\phi_{l}(1) \phi_{r}(z)\right| h_{r}\right\rangle=(1-z)^{2 \alpha_{r}\left(b+b^{-1}-\alpha_{l}\right)} \sum_{n \geq 0} z^{n} F_{n}\left(b, \alpha_{r}, \alpha_{l}, P_{r}, P_{m}, P_{l}\right),
$$

with

$$
\begin{aligned}
& F_{n}\left(b, \alpha_{r}, \alpha_{l}, P_{r}, P_{m}, P_{l}\right) \\
& \quad=\sum_{\left|Y_{1}\right|+\left|Y_{2}\right|=n} \frac{Z_{b i f}\left(\alpha_{r} \mid P_{r},(\emptyset, \emptyset) ; P_{m},\left(Y_{1}, Y_{2}\right)\right) Z_{b i f}\left(\alpha_{l} \mid P_{m},\left(Y_{1}, Y_{2}\right) ; P_{l},(\emptyset, \emptyset)\right)}{Z_{b i f}\left(0 \mid P_{m},\left(Y_{1}, Y_{2}\right) ; P_{m},\left(Y_{1}, Y_{2}\right)\right)} .
\end{aligned}
$$

Here the value of $Z_{b i f}\left(\alpha \mid P^{\prime}, \vec{Y}^{\prime} ; P, \vec{Y}\right)$ for pairs of partitions $\vec{Y}, \vec{Y}^{\prime}$ and complex numbers $P, P^{\prime}$ and $\alpha$ is given by

$$
\begin{aligned}
Z_{b i f}\left(\alpha \mid P^{\prime}, \vec{Y}^{\prime} ; P, \vec{Y}\right)= & \prod_{i, j=1}^{2}\left(\prod_{s \in Y_{i}}\left(P_{j}^{\prime}-P_{i}+b\left(l_{Y_{j}^{\prime}}(s)+1\right)-b^{-1} a_{Y_{i}}(s)-\alpha\right)\right. \\
& \left.\prod_{t \in Y_{j}^{\prime}}\left(P_{j}^{\prime}-P_{i}-b l_{Y_{i}}(t)+b^{-1}\left(a_{Y_{j}^{\prime}}(t)+1\right)-\alpha\right)\right),
\end{aligned}
$$

where, on the right hand side, $P_{i}$ is the $i$-th component of the vector $\vec{P}=(P,-P)$ and similarly for $P_{i}^{\prime}$.

5.2.4. Attempt at an estimate. A simple calculation gives

$$
\begin{aligned}
Z_{b i f} & \left(\alpha_{r} \mid P_{r},(\emptyset, \emptyset) ; P_{m},\left(Y_{1}, Y_{2}\right)\right) Z_{b i f}\left(\alpha_{l} \mid P_{m},\left(Y_{1}, Y_{2}\right) ; P_{l},(\emptyset, \emptyset)\right) \\
\quad= & \prod_{i=1}^{2} \prod_{(x, y) \in Y_{i}} \prod_{m=1}^{4}\left(\left(P_{m}\right)_{i}+b(x-1)+b^{-1}(y-1)+v_{m}\right),
\end{aligned}
$$

where $v_{1}=\alpha_{r}+P_{r}, v_{2}=\alpha_{r}-P_{r}, v_{3}=-\alpha_{l}+b+b^{-1}+P_{l}$ and $v_{4}=-\alpha_{l}+b+b^{-1}-P_{l}$. Under the identifications

$$
a_{\alpha}=P_{\alpha}, \quad \alpha=1,2, \quad \epsilon_{1}=b, \epsilon_{2}=b^{-1}, \quad w_{m}=v_{m}, \quad m=1, \ldots, 4
$$

and using Eq. (12) in Theorem 1, we recover $F_{n}\left(b, \alpha_{r}, \alpha_{l}, P_{r}, P_{m}, P_{l}\right)$ from $Z_{n}\left(\epsilon_{1}, \epsilon_{2}, \vec{a}, \vec{w} ; \lambda\right)$ in the limit $\lambda \rightarrow 0$. The conditions formulated in Theorem 5 on $b$ are $\operatorname{Re} b>0$ and either $b>0$ or $|b|=1$. The ones on $P$ are $|\operatorname{Re} P|<\frac{1}{2} \operatorname{Re} b$ and $|\operatorname{Re} P|<\frac{1}{2} \operatorname{Re} b^{-1}$. Condition (21) translates, for $\lambda=0$, to

$$
P \notin \frac{b}{2} \mathbb{Z}+\frac{b^{-1}}{2} \mathbb{Z} \text { and } b^{2} \notin \mathbb{Q}_{\geq 0} .
$$

The condition on $P$ excludes the minimal models. The conditions on $b$ restrict the central charge of the theory to the interval $(1, \infty)$. Since $\lambda^{2 r-s}=1$, Theorem 5 seems to suggest that the conformal block $\left\langle h_{l}\left|\phi_{l}(1) \phi_{r}(z)\right| h_{r}\right\rangle$ is analytic in $z$ for $|z|<1$. However, we neither know that the convergence of the Nekrasov partition function is uniform in $\lambda$, nor do we know that the convergence $Z_{n}\left(\epsilon_{1}, \epsilon_{2}, \vec{a}, \vec{w} ; \lambda\right) \rightarrow F_{n}\left(b, \alpha_{r}, \alpha_{l}, P_{r}, P_{m}, P_{l}\right)$ as $\lambda \rightarrow 0$ is uniform in $n$. 
Acknowledgements. We thank Mikhail Bershtein, Christoph Keller, Sara Pasquetti, and Jörg Teschner for useful comments and explanations. The authors were partially supported by the National Centre of Competence in Research "SwissMAP - The Mathematics of Physics" of the Swiss National Science Foundation.

Open Access This article is distributed under the terms of the Creative Commons Attribution 4.0 International License (http://creativecommons.org/licenses/by/4.0/), which permits unrestricted use, distribution, and reproduction in any medium, provided you give appropriate credit to the original author(s) and the source, provide a link to the Creative Commons license, and indicate if changes were made.

\section{Appendix A. Evaluation by iterated residues}

In this section we prove Theorem 1 by evaluating the integral by residues. In a first step, we find the position of all poles whose residues contribute to the integral and show that they are simple. In a second step we evaluate the residues.

We show that $\mathcal{Z}_{n}(\vec{u} ; \vec{p})$ is a sum

$$
\mathcal{Z}_{n}(\vec{u} ; \vec{p})=\sum_{|\vec{Y}|=n} \mathcal{Z}_{\vec{Y}}(\vec{u} ; \vec{p}),
$$

of iterated, simple residues

$$
\begin{gathered}
\mathcal{Z}_{\vec{Y}}(\vec{u} ; \vec{p}):=\left(\frac{1-q_{1} q_{2}}{\left(1-q_{1}\right)\left(1-q_{2}\right)}\right)^{n} \prod_{j=1}^{n} \prod_{m=1}^{s}\left(\hat{z}_{j}-p_{m}\right) \\
\lim _{\substack{z_{j} \rightarrow \hat{z}_{j} \\
j=1, \ldots, n}}\left(\prod_{j=1}^{n}\left(z_{j}-\hat{z}_{j}\right) \frac{\mathcal{I}\left(z_{1}, \ldots, z_{n} ; \vec{u}\right)}{z_{1} \cdots z_{n}}\right),
\end{gathered}
$$

where

$$
\left\{\hat{z}_{1}, \ldots, \hat{z}_{n}\right\}=\left\{z_{x, y}^{\alpha}:(x, y) \in Y_{\alpha}, \alpha=1, \ldots, r\right\}
$$

in any order.

We suppose that $\left|q_{1}\right|=\left|q_{2}\right|+\delta<1$, where $\delta>0$ is small enough so that $\left|q_{2}\right|>\left|q_{1}\right|^{2}>\left|q_{1}\right|^{3}>\cdots$. The general case follows from analytic continuation.

We evaluate the integral $\mathcal{Z}_{n}(\vec{u} ; \vec{p})$ by taking iterated residues. We start with a slightly more general integral. Let $U, W$ be two finite sets of complex numbers and $f\left(z_{1}, \ldots, z_{n}\right)$ a symmetric function, analytic on the closed ball in $\mathbb{C}^{n}$ with radius $\rho$. Our integral is of the form

$$
\begin{gathered}
\int_{C_{\rho}} \frac{d z_{n}}{2 \pi i} \cdots \int_{C_{\rho}} \frac{d z_{1}}{2 \pi i} I\left(z_{1}, \ldots, z_{n}\right) \\
=\int_{C_{\rho}} \frac{d z_{n}}{2 \pi i} \frac{\prod_{w \in W}\left(z_{n}-w\right)}{\prod_{u \in U}\left(z_{n}-u\right)} \prod_{n<k} \frac{\left(z_{n}-z_{k}\right)^{2}\left(z_{n}-q_{1} q_{2} z_{k}\right)\left(z_{n}-q_{1}^{-1} q_{2}^{-1} z_{k}\right)}{\left(z_{n}-q_{1} z_{k}\right)\left(z_{n}-q_{2} z_{k}\right)\left(z_{n}-q_{1}^{-1} z_{k}\right)\left(z_{n}-q_{2}^{-1} z_{k}\right)} \\
\vdots \\
\int_{C_{\rho}} \frac{d z_{2}}{2 \pi i} \frac{\prod_{w \in W}\left(z_{2}-w\right)}{\prod_{u \in U}\left(z_{2}-u\right)} \prod_{2<k} \frac{\left(z_{2}-z_{k}\right)^{2}\left(z_{2}-q_{1} q_{2} z_{k}\right)\left(z_{2}-q_{1}^{-1} q_{2}^{-1} z_{k}\right)}{\left(z_{2}-q_{1} z_{k}\right)\left(z_{2}-q_{2} z_{k}\right)\left(z_{2}-q_{1}^{-1} z_{k}\right)\left(z_{2}-q_{2}^{-1} z_{k}\right)} \\
\int_{C_{\rho}} \frac{d z_{1}}{2 \pi i} \frac{\prod_{w \in W}\left(z_{1}-w\right)}{\prod_{u \in U}\left(z_{1}-u\right)} \prod_{1<k} \frac{\left(z_{1}-z_{k}\right)^{2}\left(z_{1}-q_{1} q_{2} z_{k}\right)\left(z_{1}-q_{1}^{-1} q_{2}^{-1} z_{k}\right)}{f\left(z_{1}, \ldots, z_{1} z_{k}\right)\left(z_{1}-q_{2} z_{k}\right)\left(z_{1}-q_{1}^{-1} z_{k}\right)\left(z_{1}-q_{2}^{-1} z_{k}\right)}
\end{gathered}
$$

where $I\left(z_{1}, \ldots, z_{n}\right)$ is given by

$$
f\left(z_{1}, \ldots, z_{n}\right) \prod_{j=1}^{n}\left(\frac{\prod_{w \in W}\left(z_{j}-w\right)}{\prod_{u \in U}\left(z_{j}-u\right)}\right.
$$




$$
\left.\times \prod_{j<k} \frac{\left(z_{j}-z_{k}\right)^{2}\left(z_{j}-q_{1} q_{2} z_{k}\right)\left(z_{j}-q_{1}^{-1} q_{2}^{-1} z_{k}\right)}{\left(z_{j}-q_{1} z_{k}\right)\left(z_{j}-q_{2} z_{k}\right)\left(z_{j}-q_{1}^{-1} z_{k}\right)\left(z_{j}-q_{2}^{-1} z_{k}\right)}\right) .
$$

When integrating $z_{1}$ we can only pick up residues at $q_{1} z_{j}, q_{2} z_{j}$ or $\hat{z}_{1}=u$, for some $u \in U$ with $|u| \leq \rho$.

Assume that we pick up a residue at $\hat{z}_{1}=q_{i} z_{j}$ for some $i \in\{1,2\}$ and $j \in\{2, \ldots, n\}$. The other case will be treated later on. Let $s \in\{1,2\}$ be the index complementary to $i$. The residue is simple. After renaming $z_{j} \leftrightarrow z_{2}$ we obtain

$$
\begin{aligned}
& \int_{C_{\rho}} \frac{d z_{n}}{2 \pi i} \ldots \int_{C_{\rho}} \frac{d z_{2}}{2 \pi i} f\left(q_{i} z_{2}, z_{2}, \ldots, z_{n}\right) \\
& \quad \times \prod_{j=3}^{n}\left(\frac{\prod_{w \in W}\left(z_{j}-w\right)}{\prod_{u \in U}\left(z_{j}-u\right)} \prod_{2<j<k} \frac{\left(z_{j}-z_{k}\right)^{2}\left(z_{j}-q_{1} q_{2} z_{k}\right)\left(z_{j}-q_{1}^{-1} q_{2}^{-1} z_{k}\right)}{\left(z_{j}-q_{1} z_{k}\right)\left(z_{j}-q_{2} z_{k}\right)\left(z_{j}-q_{1}^{-1} z_{k}\right)\left(z_{j}-q_{2}^{-1} z_{k}\right)}\right) \\
& \quad \times \frac{\prod_{w \in W}\left(z_{2}-w\right)\left(q_{i} z_{2}-w\right)}{\prod_{u \in U}\left(z_{2}-u\right)\left(q_{i} z_{2}-u\right)} \\
& \times \prod_{2<k} \frac{\left(z_{2}-z_{k}\right)\left(z_{2}-q_{i} q_{s} z_{k}\right)\left(z_{2} q_{i}-z_{k}\right)\left(z_{2} q_{i}-q_{i}^{-1} q_{s}^{-1} z_{k}\right)}{\left(z_{2}-q_{i} z_{k}\right)\left(z_{2}-q_{s}^{-1} z_{k}\right)\left(z_{2} q_{i}-q_{s} z_{k}\right)\left(z_{2} q_{i}-q_{i}^{-1} z_{k}\right)} \\
& \times q_{i} z_{2} \frac{\left(q_{i}-1\right)^{2}\left(1-q_{s}\right)\left(q_{i}-q_{i}^{-1} q_{s}^{-1}\right)}{\left(q_{i}-q_{s}\right)\left(q_{i}-q_{i}^{-1}\right)\left(q_{i}-q_{s}^{-1}\right)} .
\end{aligned}
$$

We also have used Fubini's theorem to permute the order of integration, swapping the integrals over $d z_{2}$ and $d z_{j}$. This is permitted since the integrand does not have poles on the integration contours. Here we use the small perturbation of $\left|q_{1}\right|$ and $\left|q_{2}\right|$. We note that this result does not depend on $j$ anymore, which also follows from the symmetry of $I\left(z_{1}, \ldots, z_{n}\right)$. So we can suppose without loss of generality $j=2$.

We see that now we can pick up residues at $\hat{z}_{2}=u_{0}, q_{i}^{-1} u_{0}$, for some $u_{0}$ such that $\hat{z}_{2}$ lies inside the integration contour or at $\hat{z}_{2}=q_{i} z_{j}$, where the $i$ is the same as in the previous integral. It appears like there is a residue coming from the pole $\left(z_{2} q_{i}-q_{s} z_{j}\right)$ if $i=1$. However, all residues coming from these poles cancel each other in the sum: One directly calculates

$$
\lim _{z_{k} \rightarrow q_{1}^{-1} q_{2} z_{l}}\left(z_{k}-q_{1}^{-1} q_{2} z_{l}\right) \sum_{i=1,2} \sum_{j=2}^{n} \operatorname{Res}_{\hat{z}_{1}=q_{i} z_{j}} I\left(z_{1}, \ldots, z_{n}\right)=0 .
$$

Assume that we pick $\hat{z}_{2}=q_{i} z_{j}$, again assume without loss of generality $j=3$. The residue is simple. The integral over $z_{3}$ becomes

$$
\begin{aligned}
& \int_{C_{\rho}} \frac{d z_{3}}{2 \pi i} \prod_{l=0}^{2} \frac{\prod_{w \in W}\left(z_{3} q_{i}^{l}-w\right)}{\prod_{u \in U}\left(z_{3} q_{i}^{l}-u\right)} \\
& \prod_{3<k} \frac{\left(z_{3}-z_{k}\right)\left(z_{3}-q_{i} q_{s} z_{k}\right)\left(z_{3} q_{i}^{2}-z_{k}\right)\left(z_{3} q_{i}^{2}-q_{i}^{-1} q_{s}^{-1} z_{k}\right)}{\left(z_{3}-q_{i} z_{k}\right)\left(z_{3}-q_{s}^{-1} z_{k}\right)\left(z_{3} q_{i}^{2}-q_{s} z_{k}\right)\left(z_{3} q_{i}^{2}-q_{i}^{-1} z_{k}\right)} \\
& q_{i}^{3} z_{3}^{2} \frac{\left(q_{i}-1\right)^{3}\left(1-q_{s}\right)^{2}\left(q_{i}^{2}-q_{s}^{-1}\right)\left(q_{i}^{3}-q_{s}^{-1}\right)}{\left(q_{i}-q_{s}\right)\left(q_{i}-q_{s}^{-1}\right)^{2}\left(q_{i}^{2}-q_{s}\right)\left(q_{i}^{3}-1\right)} f\left(q_{i}^{2} z_{3}, q_{i} z_{3}, z_{3}, \ldots, z_{n}\right) .
\end{aligned}
$$

In the next step, we can again only pick up simple residues at $\hat{z}_{3}=q_{i} z_{4}$ or $\hat{z}_{3}=q_{i}^{-l} u_{0}$ with $l \in\{0,1,2\}$ and $u_{0} \in U$ such that $\hat{z}_{3}$ lies inside the integration contour.

Assume that we have picked and evaluated simple residues at $\left(\hat{z}_{1}, \ldots, \hat{z}_{J-1}\right)$ during the first $J-1$ integrations with $\hat{z}_{j}=q_{i}^{J-j} z_{J}, j=1, \ldots, J-1$, where $i \in\{1,2\}$ is fixed. Let $s \in\{1,2\}$ be the other index. The integral over $z_{J}$ has the integrand

$$
\prod_{l=0}^{J-1} \frac{\prod_{w \in W}\left(z_{J} q_{i}^{l}-w\right)}{\prod_{u \in U}\left(z_{J} q_{i}^{l}-u\right)}
$$




$$
\begin{aligned}
& \times \prod_{J<k} \frac{\left(z_{J}-z_{k}\right)\left(z_{J}-q_{i} q_{s} z_{k}\right)\left(z_{J} q_{i}^{J-1}-z_{k}\right)\left(z_{J} q_{i}^{J-1}-q_{i}^{-1} q_{s}^{-1} z_{k}\right)}{\left(z_{J}-q_{i} z_{k}\right)\left(z_{J}-q_{s}^{-1} z_{k}\right)\left(z_{J} q_{i}^{J-1}-q_{s} z_{k}\right)\left(z_{J} q_{i}^{J-1}-q_{i}^{-1} z_{k}\right)} \\
& \times z_{J}^{J-1} q_{i}^{\frac{1}{2}(J+2)(J-1)} \frac{\left(q_{i}-1\right)^{J}\left(1-q_{s}\right)^{J-1}}{\left(q_{i}-q_{s}^{-1}\right)^{J-1}\left(q_{i}^{J}-1\right)} \\
& \times \prod_{j=1}^{J-1} \frac{\left(q_{i}^{j}-q_{i}^{-1} q_{s}^{-1}\right)}{\left(q_{i}^{j}-q_{s}\right)} f\left(q_{i}^{J-1} z_{J}, \ldots, z_{J}, \ldots, z_{n}\right),
\end{aligned}
$$

whereas the integrands for $z_{J+1}, \ldots, z_{n}$ in Eq. (32) remain the same. Now pick a residue at $\hat{z}_{J}=q_{i}^{-l_{0}} u_{0}$ inside the integration contour with $u_{0} \in U$ and $l_{0} \in\{0, \ldots, J-1\}$ and suppose the pole is simple. So we have followed the evaluation steps

$$
\hat{z}_{1}=q_{i} z_{2}, \quad \hat{z}_{2}=q_{i} z_{3}, \quad \ldots, \quad \hat{z}_{J-1}=q_{i} z_{J}, \quad \hat{z}_{J}=q_{i}^{-l_{0}} u_{0}
$$

leading to the residue strip

$$
\left(\hat{z}_{1}, \ldots, \hat{z}_{J}\right)=q_{i}^{-l_{0}}\left(q_{i}^{J-1} u_{0}, \ldots, u_{0}\right)
$$

When we evaluate the final residue we get the same integral expression we started with as in Eq. (32), except that we only have the variables $z_{J+1}, \ldots, z_{n}$, the sets $U, W$ are changed to

$$
U^{\prime}=U \cup\left\{q_{i}^{-1} \hat{z}_{J}, q_{s} \hat{z}_{J}, q_{s}^{-1} \hat{z}_{1}, q_{i} \hat{z}_{1}\right\} \quad W^{\prime}=W \cup\left\{\hat{z}_{J}, q_{i}^{-1} q_{s}^{-1} \hat{z}_{J}, \hat{z}_{1}, q_{i} q_{s} \hat{z}_{1}\right\},
$$

and $f\left(z_{1}, \ldots, z_{n}\right)$ gets replaced by

$$
f^{\prime}\left(z_{J+1}, \ldots, z_{n}\right)=f\left(q_{i}^{J-1} q_{i}^{-l_{0}} u_{0}, \ldots, q_{i}^{-l_{0}} u_{0}, z_{J+1}, \ldots, z_{n}\right) .
$$

This function is again symmetric. Moreover, we have accumulated a prefactor with the value

$$
\begin{aligned}
& \prod_{l=0}^{J-1} \frac{\prod_{w \in W}\left(q_{i}^{l-l_{0}} u_{0}-w\right)}{\prod_{u \in U \backslash\left\{u_{0}\right\}}\left(q_{i}^{l-l_{0}} u_{0}-u\right)} \prod_{\substack{l=0 \\
l \neq l_{0}}}^{J-1} \frac{1}{\left(q_{i}^{l-l_{0}}-1\right)} q_{i}^{\frac{1}{2(J+2)(J-1)-l_{0} J}} \\
& \frac{\left(q_{i}-1\right)^{J}\left(1-q_{s}\right)^{J-1}}{\left(q_{i}-q_{s}^{-1}\right)^{J-1}\left(q_{i}^{J}-1\right)} \prod_{j=1}^{J-1} \frac{\left(q_{i}^{j}-q_{i}^{-1} q_{s}^{-1}\right)}{\left(q_{i}^{j}-q_{s}\right)}
\end{aligned}
$$

coming from the residue evaluation. We see that the evaluation of our integral happens in stages, where one evaluates a strip of residues. We draw the strip (35) for $i=2$ as follows:

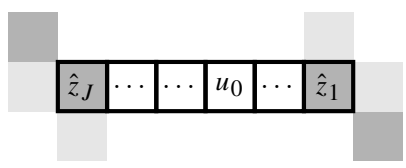

The positive $q_{i}$ direction goes from west to east. The residues go from east to west over the residue strip (35). Here we have also indicated the poles (light gray) and zeros (dark grey) such a strip adds to the sets $U$ and $W$. For $i=1$ the strip is drawn vertically, with positive $q_{i}$ direction from north to south.

All formulae remain valid for $J=1$, i.e. when we directly pick up a residue at $\hat{z}_{1}=u_{0}$ for some $u_{0} \in U$. We call such a strip of length one a box. It is drawn as

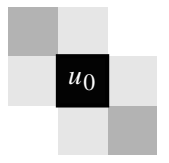

Here we have used black to mark a zero of order two. We want to compare the result of the evaluation process (34) of the above residues for general $l_{0} \in\{0, \ldots, J-1\}$ to the result from the procedure where we evaluate 
the same final residues (35) by repeated use of the case $J=1$. Now, the order of the residues will be different. It is clear that the sets $U^{\prime}$ and $W^{\prime}$ we end up with agree for both procedures. Moreover, the new symmetric function $f^{\prime}$ also agrees, since the original function $f$ is symmetric. However the prefactor (36) only agrees up to a sign, as we will now see.

We first treat the case, where we go in positive $q_{i}$ direction, starting at a pole at some $u_{1}$ : Suppose we pick up residues using the evaluation process

$$
\hat{z}_{1}=u_{1}, \quad \hat{z}_{2}=q_{i} u_{1}, \quad \ldots, \quad \hat{z}_{K}=q_{i}^{K-1} u_{1} .
$$

For $k=0, \ldots, K$, let $U_{k}^{+}$and $W_{k}^{+}$denote the sets $U$ and $W$ after evaluating the residues at $\hat{z}_{1}, \ldots, \hat{z}_{k}$. In the first step we pick up the residue at $\hat{z}_{1}=u_{1}$, the sets $U=U_{0}^{+}$and $W=W_{0}^{+}$get changed to

$$
\begin{aligned}
U_{1}^{+} & =\left(U \backslash\left\{u_{1}\right\}\right) \cup\left\{q_{i}^{-1} u_{1}, q_{s} u_{1}, q_{s}^{-1} u_{1}, q_{i} u_{1}\right\} \\
W_{1}^{+} & =W \cup\left\{u_{1}, q_{i}^{-1} q_{s}^{-1} u_{1}, q_{i} q_{s} u_{1}\right\},
\end{aligned}
$$

the symmetric function is $f\left(u_{1}, z_{2}, \ldots, z_{n}\right)$ and we get the prefactor

$$
\frac{\prod_{w \in W_{0}^{+}}\left(u_{1}-w\right)}{\prod_{u \in U_{0}^{+} \backslash\left\{u_{1}\right\}}\left(u_{1}-u\right)} .
$$

By induction, we see that after evaluating all residues (39), we have changed the original sets $U, W$ to

$$
\begin{aligned}
U_{K}^{+} & =\left(U \backslash\left\{u_{1}\right\}\right) \cup\left\{q_{i}^{-1} u_{1}, q_{s} u_{1}, q_{s}^{-1} q_{i}^{K-1} u_{1}, q_{i} q_{i}^{K-1} u_{1}\right\} \\
W_{K}^{+} & =W \cup\left\{q_{i}^{-1} q_{s}^{-1} u_{1}, q_{i}^{K-1} u_{1}, q_{i} q_{s} q_{i}^{K-1} u_{1}\right\},
\end{aligned}
$$

the function $f\left(z_{1}, \ldots, z_{n}\right)$ gets replaced by

$$
f_{K}^{+}\left(z_{K+1}, \ldots, z_{n}\right)=f\left(u_{1}, \ldots, q_{i}^{K-1} u_{1}, z_{K+1}, \ldots, z_{n}\right)
$$

and we have accumulated the prefactor

$$
\begin{aligned}
& \prod_{k=0}^{K-1} \frac{\prod_{w \in W_{k}^{+}}\left(q_{i}^{k} u_{1}-w\right)}{\prod_{u \in U_{k}^{+} \backslash\left\{q_{i}^{k} u_{1}\right\}}\left(q_{i}^{k} u_{1}-u\right)}=\prod_{k=0}^{K-1} \frac{\prod_{w \in W}\left(q_{i}^{k} u_{1}-w\right)}{\prod_{u \in U \backslash\left\{u_{1}\right\}}\left(q_{i}^{k} u_{1}-u\right)} \prod_{k=1}^{K-1} \frac{1}{\left(q_{i}^{k}-1\right)} \\
& q_{i}^{\frac{1}{2}(K+2)(K-1)} \frac{\left(q_{i}-1\right)^{K}\left(1-q_{s}\right)^{K-1}}{\left(q_{i}^{K}-1\right)\left(q_{i}-q_{s}^{-1}\right)^{K-1}} \prod_{k=1}^{K-1} \frac{\left(q_{i}^{k}-q_{i}^{-1} q_{s}^{-1}\right)}{\left(q_{i}^{k}-q_{s}\right)} .
\end{aligned}
$$

Next, we treat the case where we go in negative $q_{i}$ direction starting at some pole $u_{2}$. Suppose we pick up residues using the evaluation steps

$$
\hat{z}_{1}=u_{2}, \quad \hat{z}_{2}=q_{i}^{-1} u_{2}, \quad \ldots, \quad \hat{z}_{M}=q_{i}^{-M+1} u_{2} .
$$

For $m=0, \ldots, M$, let $U_{m}^{-}$and $W_{m}^{-}$denote the sets $U$ and $W$ after evaluating the residues at $\hat{z}_{1}, \ldots, \hat{z}_{m}$. In the first step we pick up the residue at $\hat{z}_{1}=u_{2}$, the sets $U=U_{0}^{-}$and $W=W_{0}^{-}$get changed to

$$
\begin{aligned}
U_{1}^{-} & =\left(U \backslash\left\{u_{2}\right\}\right) \cup\left\{q_{i}^{-1} u_{2}, q_{s} u_{2}, q_{s}^{-1} u_{2}, q_{i} u_{2}\right\} \\
W_{1}^{-} & =W \cup\left\{u_{2}, q_{i}^{-1} q_{s}^{-1} u_{2}, q_{i} q_{s} u_{2}\right\},
\end{aligned}
$$

the symmetric function is $f\left(u_{2}, z_{2}, \ldots, z_{n}\right)$ and we get the prefactor

$$
\frac{\prod_{w \in W_{0}^{-}}\left(u_{2}-w\right)}{\prod_{u \in U_{0}^{-} \backslash\left\{u_{2}\right\}^{(}}\left(u_{2}-u\right)} .
$$

Again, after evaluating all residues (41), we have changed the original sets $U, W$ to

$$
U_{M}^{-}=\left(U \backslash\left\{u_{2}\right\}\right) \cup\left\{q_{i} u_{2}, q_{s}^{-1} u_{2}, q_{s} q_{i}^{-M+1} u_{2}, q_{i}^{-1} q_{i}^{-M+1} u_{2}\right\}
$$




$$
W_{M}^{-}=W \cup\left\{q_{i} q_{s} u_{2}, q_{i}^{-M+1} u_{2}, q_{i}^{-1} q_{s}^{-1} q_{i}^{-M+1} u_{2}\right\},
$$

the function $f\left(z_{1}, \ldots, z_{n}\right)$ is replaced by

$$
f_{M}^{-}\left(z_{M+1}, \ldots, z_{n}\right)=f\left(u_{2}, \ldots, q_{i}^{-M+1} u_{2}, z_{M+1}, \ldots, z_{n}\right)
$$

and get the prefactor

$$
\begin{aligned}
& \prod_{m=0}^{M-1} \frac{\prod_{w \in W_{m}^{-}}\left(q_{i}^{-m} u_{2}-w\right)}{\prod_{u \in U_{m}^{-} \backslash\left\{q_{i}^{-m} u_{2}\right\}}\left(q_{i}^{-m} u_{2}-u\right)}=\prod_{m=0}^{M-1} \frac{\prod_{w \in W}\left(q_{i}^{-m} u_{2}-w\right)}{\prod_{u \in U \backslash\left\{u_{2}\right\}}\left(q_{i}^{-m} u_{2}-u\right)} \prod_{m=1}^{M-1} \frac{1}{\left(q_{i}^{-m}-1\right)} \\
& q_{i}^{-\frac{1}{2}(M-1) M+M-1} \frac{\left(1-q_{i}\right)^{M}\left(1-q_{s}\right)^{M-1}}{\left(q_{i}-q_{s}^{-1}\right)^{M-1}\left(1-q_{i}^{M}\right)} \prod_{m=1}^{M-1} \frac{\left(q_{i}^{m}-q_{i}^{-1} q_{s}^{-1}\right)}{\left(q_{i}^{m}-q_{s}\right)} .
\end{aligned}
$$

Recall that were looking at the case where we pick the residues (35) as a strip depicted in figure (37) using the evaluation steps (34) and we want to compare it to the procedure where we apply the case $J=1$ repeatedly. Set $M=l_{0}+1$ and $K=J-l_{0}-1$. Picking residues according to the stepwise procedures with $u_{2}=u_{0}$ and $u_{1}=q_{i} u_{0}$ we get the prefactor

$$
\begin{aligned}
& \prod_{k=0}^{K-1} \frac{\prod_{w \in W_{M}^{-}}\left(q_{i}^{k} u_{1}-w\right)}{\prod_{u \in U_{M}^{-} \backslash\left\{u_{1}\right\}}\left(q_{i}^{k} u_{1}-u\right)} \prod_{m=0}^{M-1} \frac{\prod_{w \in W}\left(q_{i}^{-m} u_{2}-w\right)}{\prod_{u \in U \backslash\left\{u_{2}\right\}}\left(q_{i}^{-m} u_{2}-u\right)} \\
& q_{i}^{\frac{1}{2}(K+2)(K-1)} q_{i}^{-\frac{1}{2}(M-1) M+M-1} \frac{\left(q_{i}-1\right)^{K}\left(1-q_{s}\right)^{K-1}}{\left(q_{i}^{K}-1\right)\left(q_{i}-q_{s}^{-1}\right)^{K-1}} \frac{\left(1-q_{i}\right)^{M}\left(1-q_{s}\right)^{M-1}}{\left(q_{i}-q_{s}^{-1}\right)^{M-1}\left(1-q_{i}^{M}\right)} \\
& \prod_{k=1}^{K-1} \frac{\left(q_{i}^{k}-q_{i}^{-1} q_{s}^{-1}\right)}{\left(q_{i}^{k}-q_{s}\right)} \prod_{m=1}^{M-1} \frac{\left(q_{i}^{m}-q_{i}^{-1} q_{s}^{-1}\right)}{\left(q_{i}^{m}-q_{s}\right)} \prod_{k=1}^{K-1} \frac{1}{\left(q_{i}^{k}-1\right)} \prod_{m=1}^{M-1} \frac{1}{\left(q_{i}^{-m}-1\right)}
\end{aligned}
$$

We call this factor the base value for our strip of residues. This factor equals the prefactor in Eq. (36) up to a sign of

$$
(-1)^{M+1}
$$

(When we first evaluate the residues in positive $q_{i}$ direction and then in negative $q_{i}$ direction we get the same result.)

We point out two special cases of our observation:

(1) In the case $l_{0}=0$, where we place the whole strip of residues at $u_{0}$ and eastwards of $u_{0}$, it does not matter whether we pick the residues one by one or as a strip.

(2) In the case $l_{0}=J-1$, where we place the whole strip of residues at $u_{0}$ and westwards of $u_{0}$, we get the base value up to a sign equal to $(-1)^{L+1}$ where $L$ is the length of the strip.

Claim 1. Only strips with $l_{0}=0$ contribute to the integral.

Suppose, we add a strip of residues of length $J$ with $l_{0}>0$, i.e. part of the residue diagram grows in negative $q_{i}$ direction. We claim that the total contribution of all possible processes to choose residues leading to the same residue strip is zero. We depict the strip as

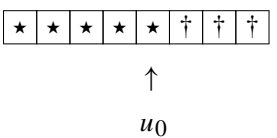

for $i=2$ and vertically for $i=1$. The number of stars equals $M=l_{0}+1$ and the number of daggers equals $K=J-M$. The residue at $u_{0}$ is located at the easternmost star. We draw the corresponding base value, corresponding to a stepwise procedure when picking up residues, as 
Of course, this picture does not fully specify the procedure: We can alternate between picking up residues to the east and to the west. We will deal with this multiplicity later.

Another way to evaluate the residues is for example by first evaluating some of the residues as a strip with $l_{0}=J-2$ and then two as boxes and one final strip of length two to the east. We depict this as

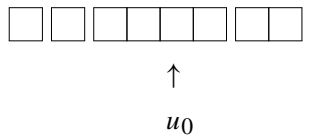

All possible ways to end up with the residues (35) define a partition of the strip (44) into substrips. All those partitions yield the same final sets $U^{\prime}, W^{\prime}$ and the same final function $f^{\prime}$. The contributions of the residue evaluations differ by signs depending on the partition into substrips. To compute these signs we have to cut the partition eastwards of $u_{0}$, for example we substitute the partition (45) by

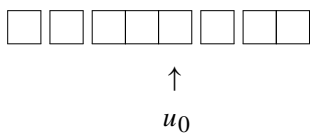

According to our computation above, we then have to count the number of strips of even length westwards of $u_{0}$. For each of those, we get a factor of $(-1)$. Then we have to sum over all partitions of the original string, weighted by the number of ways in which one can pick residues to obtain the partition. As we have already noticed, there are multiple ways since one can for example alternate between placing substrips at the west and east end. The total contribution of all procedures leading to the final residues (35) is given by the base value times an expression

$$
\sum_{\xi \in \Gamma} w(\xi) s(\xi)
$$

which we want to formalize. Here the set $\Gamma$ represents all the ways to cut the strip (44) into substrips. The integer $w(\xi)$ is the weight corresponding to all possible procedures to pick residues leading to the choice of substrips $\xi$. Finally the $\operatorname{sign} s(\xi)$ is given by the number of substrips of even length to the west of $u_{0}$ after cutting directly to the east of $u_{0}$. We set

$$
\Gamma_{J}=\left\{\left(J_{1}, \ldots, J_{N}\right): N \in \mathbb{N}, J_{1}, \ldots, J_{N} \in \mathbb{N}: J_{0}+\cdots+J_{N}=J\right\}
$$

to be the set of ordered partitions of the integer $J$. Cutting the strip (44) into substrips according to $\vec{J} \in \Gamma J$ means cutting it into substrips of length $J_{0}, \ldots, J_{n}$ from west to east. For example the cut strip (45) corresponds to the element $\vec{J}=(1,1,4,2) \in \Gamma_{8}$. Let

$$
\text { cut }: \Gamma_{J} \rightarrow \Gamma_{M}
$$

be the map realized by cutting the collection of substrips $\vec{J}$ directly to the east of $u_{0}$. Now we can formalize the $\operatorname{sign} s(\vec{J})$ as

$$
s(\vec{J})=\prod_{a}(-1)^{1+(\operatorname{cut}(\vec{J}))_{a} .}
$$

In order to describe the weight $w(\vec{J})$, we have to take into account two effects: Firstly, for each substrip of length $J_{a}>1$, we have to count the number of choices when selecting a residue $\hat{z}_{a}=q_{i} z_{b}$ that correspond to the freedom to choose any of the remaining variables $z_{b}$ for $b \in\{1, \ldots, J\}$. Secondly, we can alternate between placing substrips to the west of the already chosen residues or to the east of them. We first have to account for the second effect. To model the second effect, we reorder the vectors $\vec{J}$. We define the map

$$
\text { reo }: \Gamma_{J} \rightarrow \Gamma_{J}
$$

by demanding that, for

$$
\vec{A}=\left(A_{1}, A_{2}, \ldots, A_{N}\right)=\operatorname{reo}(\vec{J})=\left(B_{0}, B_{1}, \ldots, B_{b}, C_{1}, \ldots, C_{c}\right),
$$


the number $B_{0}$ corresponds to the substrip containing $u_{0}$, the numbers $B_{1}$ up to $B_{b}$ correspond to the substrips to the west, with increasing index in westward direction, and the numbers $C_{1}$ up to $C_{c}$ correspond to substrips to the east, with increasing index in eastward direction. The indices $b=b(\vec{J})$ and $c=c(\vec{J})$ depend on the collection of substrips $\vec{J} \in \Gamma_{J}$. For example, the cut strip (45) yields $\operatorname{reo}(\vec{J})=(4,1,1,2), b=2$ and $c=1$. We interpret the order of the components of $\operatorname{reo}(\vec{J})$ as the order in which we place the residues. All other orderings are obtained by permuting the elements $(\vec{B}, \vec{C})=\left(B_{1}, \ldots, B_{b}, C_{1}, \ldots, C_{c}\right)$ of this tuple, such that the order of the elements in $\vec{B}$ and in $\vec{C}$ remain the same. This means we can alternate between placing residues to the west and to the east. Let

$$
S_{b, c}=\left\{\sigma \in S_{b+c}: \sigma(1)<\cdots<\sigma(b), \sigma(b+1)<\cdots<\sigma(b+c)\right\}
$$

denote the set of $(b, c)$ shuffles. We define

$$
\sigma \vec{A}=\sigma\left(B_{0}, B_{1}, \ldots, B_{b}, C_{1}, \ldots, C_{c}\right)=\left(B_{0}, \sigma\left(B_{1}, \ldots, B_{b}, C_{1}, \ldots, C_{c}\right)\right),
$$

where $\sigma$ acts on $\left(B_{1}, \ldots, B_{b}, C_{1}, \ldots, C_{c}\right)$ in the usual sense. We see that all possible ways to place the substrips reo $(\vec{J})$ corresponds to the orbit of this tuple under $S_{r, s}$. Hence we have formalized the second effect. Now we can also formalize the first effect described above: Suppose we are placing the substrip $\sigma(\vec{A})_{k}$. Then the remaining substrips correspond to $\sigma(\vec{A})_{k+1}+\cdots+\sigma(\vec{A})_{N}$ variables. Each time we choose a pole at $\hat{z}_{a}=q_{i} z_{b}$ in $\sigma(\vec{A})_{k}$, we can choose $z_{b}$ to be one of those variables, or one of the variables in $\sigma(\vec{A})_{k}$, we have not chosen so far. Hence, we get

$$
\prod_{h=1}^{\sigma(\vec{A})_{j}-1}\left(\sum_{k=j+1}^{N} \sigma(\vec{A})_{k}+h\right)
$$

possibilities in total when placing $\sigma(\vec{A})_{k}$. In the case $\sigma(\vec{A})_{k}=1$, we get an empty product which we interpret as one. This agrees with the observation that $\sigma(\vec{A})_{k}=1$ implies we pick a residue from the set $U$, which leaves no further choices in terms of the remaining variables. Now we can combine the two effects to obtain the expression

$$
w(\vec{J})=\sum_{\sigma \in S_{b, c}} \prod_{j=1}^{N}\left(\prod_{h=1}^{\sigma(\vec{A})_{j}-1}\left(\sum_{k=j+1}^{N} \sigma(\vec{A})_{k}+h\right)\right)
$$

where $b=b(\vec{J}), c=c(\vec{J})$ and $\vec{A}=\operatorname{reo}(\vec{J})$. In order to prove claim 1 , we show

$$
\begin{aligned}
& \sum_{\vec{J} \in \Gamma_{J}} s(\vec{J}) w(\vec{J})=\sum_{\vec{J} \in \Gamma_{J}}\left(\prod_{a}(-1)^{\left.1+(\operatorname{cut}(\vec{J}))_{a}\right)}\right. \\
& \quad \times\left(\sum_{\sigma \in S_{b(\vec{J}), c(\vec{J})}} \prod_{j=1}^{N}\left(\prod_{h=1}^{\sigma(\operatorname{reo}(\vec{J}))_{j}-1}\left(\sum_{k=j+1}^{N} \sigma(\operatorname{reo}(\vec{J}))_{k}+h\right)\right)\right)=0
\end{aligned}
$$

by exhibiting parts of this sum that cancel each other. Fix one $\vec{J} \in \Gamma_{J}$ with $B_{b}>1$ for

$$
\vec{A}=\operatorname{reo}(\vec{J})=\left(B_{0}, B_{1}, \ldots, B_{b-1}, B_{b}, C_{1}, \ldots, C_{c}\right)
$$

We compare this choice of substrips to

$$
\begin{aligned}
\vec{A}^{\prime} & :=\left(B_{0}, B_{1}, \ldots, B_{b-1}, B_{b}-1,1, C_{1}, \ldots, C_{c}\right) \\
& =:\left(B_{0}^{\prime}, B_{1}^{\prime}, \ldots, B_{b+1}^{\prime}, C_{1}^{\prime}, \ldots, C_{c}^{\prime}\right)
\end{aligned}
$$

Graphically, $\vec{A}^{\prime}$ is obtained from $\vec{A}$ by cutting the strip at the westernmost box. Let $\vec{J}^{\prime} \in \Gamma_{J}$ with $\vec{A}^{\prime}=\operatorname{reo}\left(\vec{J}^{\prime}\right)$. We claim that

$$
w(\vec{J}) s(\vec{J})=-w\left(\vec{J}^{\prime}\right) s\left(\vec{J}^{\prime}\right)
$$


This suffices: There is a one to one correspondence between collections of substrips whose westernmost substrip has length one and those whose westernmost substrip has length greater than one. A bijection is given by $\vec{A} \mapsto \vec{A}^{\prime}$. It is clear from the definition of $s(\vec{J})$ that $\vec{J}$ and $\vec{J}^{\prime}$ have opposite sign, provided $l_{0}>0$. Hence it suffices to show that their weight is equal. We thus want to prove the equality in

$$
\begin{aligned}
& \sum_{\sigma \in S_{b, c}} \prod_{j=1}^{N}\left(\prod_{h=1}^{\sigma(\vec{A})_{j}-1}\left(\sum_{k=j+1}^{N} \sigma(\vec{A})_{k}+h\right)\right) \\
& =\sum_{\sigma \in S_{b+1, c}} \prod_{j=1}^{N+1}\left(\prod_{h=1}^{\sigma\left(\vec{A}^{\prime}\right)_{j}-1}\left(\sum_{k=j+1}^{N+1} \sigma\left(\vec{A}^{\prime}\right)_{k}+h\right)\right) .
\end{aligned}
$$

We decompose

$$
S_{b+1, c}=\bigcup_{\sigma \in S_{b, c}}\left\{\hat{\sigma}_{\lambda}: \lambda=1, \ldots, N+1-\sigma(r)\right\}
$$

where, for $j=1, \ldots, N+1$,

$$
\hat{\sigma}_{\lambda}(j)= \begin{cases}\sigma(j), & j=1, \ldots, b \\ \sigma(b)+\lambda, & j=b+1 \\ \sigma(j-1), & j=b+2, \ldots, \sigma(b)+\lambda \\ \sigma(j-1)+1, & j>\sigma(b)+\lambda+1 .\end{cases}
$$

The parameter $\lambda$ tells us how much we delay the placement of the residue box we cut away from the westernmost residue strip. Fix $\sigma \in S_{b, c}$. We will show

$$
\begin{aligned}
& \prod_{j=1}^{N}\left(\prod_{h=1}^{\sigma(\vec{A})_{j}-1}\left(\sum_{k=j+1}^{N} \sigma(\vec{A})_{k}+h\right)\right) \\
& =\sum_{\lambda=1}^{N+1-\sigma(b)} \prod_{j=1}^{N+1}\left(\prod_{h=1}^{\hat{\sigma}_{\lambda}\left(\vec{A}^{\prime}\right)_{j}-1}\left(\sum_{k=j+1}^{N+1} \hat{\sigma}_{\lambda}\left(\vec{A}^{\prime}\right)_{k}+h\right)\right) .
\end{aligned}
$$

We set $B:=\sigma(b)$. This is step in which we place $B_{b}^{\prime}$. The tuples $\hat{\sigma}_{\lambda}\left(\vec{A}^{\prime}\right)$ and $\sigma(\vec{A})$ are related as follows:

$$
\begin{aligned}
& \left(\hat{\sigma}_{\lambda}\left(\vec{A}^{\prime}\right)_{1}, \ldots, \hat{\sigma}_{\lambda}\left(\vec{A}^{\prime}\right)_{B-1}, \hat{\sigma}_{\lambda}\left(\vec{A}^{\prime}\right)_{B}, \hat{\sigma}_{\lambda}\left(\vec{A}^{\prime}\right)_{B+1}, \ldots, \hat{\sigma}_{\lambda}\left(\vec{A}^{\prime}\right)_{N+1}\right) \\
& \quad=\left(\sigma(\vec{A})_{1}, \ldots, \sigma(\vec{A})_{B-1}, \sigma(\vec{A})_{B}-1,\left(\sigma(\vec{A})_{B+1}, \ldots, 1, \ldots, \sigma(\vec{A})_{N}\right)\right),
\end{aligned}
$$

where 1 sits at index $\lambda$ inside the inner tuple. Using this description, we rewrite the right hand side of Eq. (47) as

$$
\begin{gathered}
\sum_{\lambda=1}^{N+1-B} \prod_{j=1}^{B-1}\left(\prod_{h=1}^{\sigma(\vec{A})_{j}-1}\left(\sum_{k=j+1}^{N} \sigma(\vec{A})_{k}+h\right)\right)\left(\prod_{h=2}^{\sigma(\vec{A})_{B}-1}\left(\sum_{k=B+1}^{N} \sigma(\vec{A})_{k}+h\right)\right) \\
\prod_{j=B+1}^{B+\lambda-1}\left(\prod_{h=2}^{\sigma(\vec{A})_{j}}\left(\sum_{k=j+1}^{N} \sigma(\vec{A})_{k}+h\right)\right) \prod_{j=B+\lambda}^{N}\left(\prod_{h=1}^{\sigma(\vec{A})_{j}-1}\left(\sum_{k=j+1}^{N} \sigma(\vec{A})_{k}+h\right)\right) .
\end{gathered}
$$

This equals the left hand side of Eq. (47) up to a factor of

$$
\left(\sum_{k=B+1}^{N} \sigma(\vec{A})_{k}+1\right)^{-1} \sum_{\lambda=1}^{N+1-B} \prod_{j=B+1}^{B+\lambda-1}\left(\left(\sum_{k=j+1}^{N} \sigma(\vec{A})_{k}+1\right)^{-1}\right.
$$




$$
\left.\left(\sum_{k=j+1}^{N} \sigma(\vec{A})_{k}+\sigma(\vec{A})_{j}\right)\right) .
$$

This factor equals one, since for arbitrary tuples $\left(x_{1}, \ldots, x_{L}\right)$, we have

$$
\begin{aligned}
\sum_{J=1}^{L} & \prod_{j=1}^{J} \frac{\sum_{k=j}^{L} x_{k}}{\sum_{k=j+1}^{L} x_{k}+1} \\
= & \frac{\sum_{k=1}^{L} x_{k}}{\sum_{k=2}^{L} x_{k}+1}\left(1+\frac{\sum_{k=2}^{L} x_{k}}{\sum_{k=3}^{L} x_{k}+1}\right. \\
& \times\left(\cdots\left(1+\frac{x_{k-2}+x_{k-1}+x_{k}}{x_{k-1}+x_{k}+1}\left(1+\frac{x_{k-1}+x_{k}}{x_{k}+1}\left(1+\frac{x_{k}}{1}\right)\right) \cdots\right)\right) \\
= & \sum_{k=1}^{L} x_{k} .
\end{aligned}
$$

Hence we have established Eq. (46) and thus proved claim 1. We note two consequences of our argument up to now:

Claim 2. Since we can only consider strips with $l_{0}=0$, we can only consider the cases were we take single boxes as residues. In particular, the order of their evaluation does not matter.

Claim 3. We can therefore discard poles at $q_{i}^{-1} u_{0}$ with $u_{0} \in U$ since they are either out of the integration contours or realizable by a string with $J=2$ and $l_{0}=1$ and hence part of a zero sum described above.

Now we see inductively that the residues $\hat{z}_{j}$ contributing to the integral are of the form $u_{\alpha} q_{1}^{x-1} q_{2}^{y-1}$ where $(x, y) \in Y_{\alpha}$ for some Young diagrams $Y_{\alpha}$. Because of the condition

$$
u_{\alpha} u_{\beta}^{-1} \notin\left\{q_{1}^{x} q_{2}^{y}: x, y \in \mathbb{Z}\right\}, \quad \alpha \neq \beta
$$

the poles do not interact and we may suppose $r=1$. The sets $U$ and $W$ we start with are then $U=\left\{u_{1}\right\}$ and $W=\emptyset$. We evaluate a residue at the pole $u_{1}$ changing the zeros and poles of the remaining integrand. We depict this process as

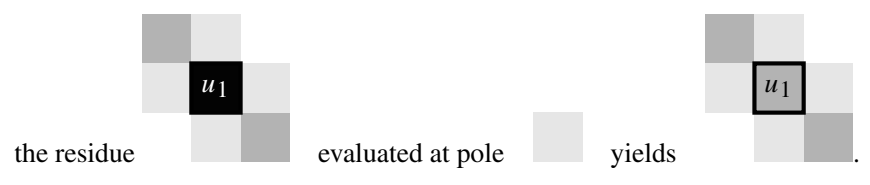

For the induction step, assume we have evaluated residues such that the evaluated residues fill a Young diagram. Also assume that the poles and zeros encoded in the sets $U, W$ are located at the following places in the diagram: at each corner outside the diagram which is open to the south east there is a pole. To the northwest of each of those poles is another pole. At all south-easternmost boxes in the diagram there is a zero and another zero directly south east of it. Finally there is a zero at the coordinate $(-1,-1)$. An example would be for instance

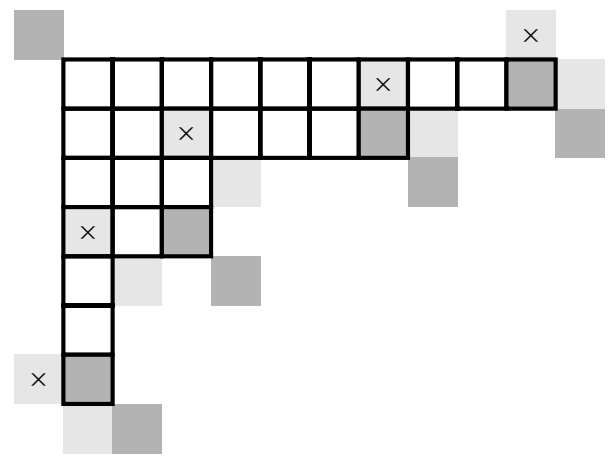


As we have seen before in claim 3, evaluating a residue at the poles marked with a cross does not contribute to the integral. It is clear that placing a residue box as in (38) at one of the remaining poles in diagram (48) yields again a Young diagram with the same structure of the poles and zeros as in diagram (48).

Hence, we have established that all residues are simple residues located at (31). It is clear that different procedures to pick residues at $\left\{z_{s}^{\alpha}: s \in Y_{\alpha}, \alpha=1, \ldots, r\right\}$ correspond to permutation of the variables $\hat{z}_{j}, j=1, \ldots, n$. Since the integral is symmetric in $z_{1}, \ldots, z_{n}$, all possible permutations occur. This cancels the factor $n$ ! in front of the integral.

We have proved Eq. (29). Next, we evaluate the right hand side of Eq. (30). The integrand (8) is invariant under swapping $q_{1}$ and $q_{2}$. The residues $\left\{z_{s}^{\alpha}: s \in Y_{\alpha}, \alpha=1, \ldots, r\right\}$ defined in Eq. (10) remain invariant if we swap $q_{1}$ and $q_{2}$ and transpose the diagrams $Y_{1}, \ldots, Y_{r}$. Hence

$$
\sum_{|\vec{Y}|=n} \mathcal{Z}_{Y_{1}, \ldots, Y_{r}}(\vec{u} ; \vec{p})=\left.\sum_{|\vec{Y}|=n} \mathcal{Z}_{Y_{1}^{T}, \ldots Y_{r}^{T}}(\vec{u} ; \vec{p})\right|_{q_{1} \leftrightarrow q_{2}}
$$

We have $a_{Y}(x, y)=l_{Y T}(y, x)$ and hence Eq. (13) follows from Eq. (12).

The proof of Eq. (12) is adapted from [24], where it was performed for the special case $r=2$. It suffices to consider $\vec{p}=\emptyset$ since we can cancel the factors in the equation we want to prove. Let

$$
\begin{aligned}
\mathcal{R}_{\vec{Y}}(\vec{u} ; \emptyset)= & \prod_{\alpha, \beta=1}^{r}\left(\prod_{s \in Y_{\alpha}} \frac{1}{1-u_{\alpha} u_{\beta}^{-1} q_{1}^{l_{Y_{\alpha}}(s)+1} q_{2}^{-a_{Y_{\beta}}(s)}}\right. \\
& \left.\prod_{t \in Y_{\beta}} \frac{1}{1-u_{\alpha} u_{\beta}^{-1} q_{1}^{-l_{Y_{\beta}}(t)} q_{2}^{a_{Y_{\alpha}}(t)+1}}\right)
\end{aligned}
$$

be the right hand side of the first equation in Theorem 1.

For any $r$-tuple $\vec{Y}$ of partitions with $Y_{r} \neq \emptyset$, define the $r$-tuple $\vec{Y}^{\prime}$ of partitions by removing the last box from the last partition in $\vec{Y}$, i.e., we set $Y_{\alpha}^{\prime}=Y_{\alpha}$ for $\alpha=1, \ldots, r-1$ and $Y_{r}^{\prime}=\left(Y_{1}, \ldots, Y_{l-1}, Y_{l}-1\right)$, where $l$ is the length of $Y_{r}$. In terms of Young diagrams, we go from $\vec{Y}$ to $\vec{Y}^{\prime}$ by removing the box

$$
(l, w):=\left(l\left(Y_{r}\right), Y_{r}(l)\right)
$$

from the last Young diagram $Y_{r}$ in $\vec{Y}$. We will prove

$$
\frac{\mathcal{Z}_{\vec{Y}}(\vec{u} ; \emptyset)}{\mathcal{Z}_{\vec{Y}^{\prime}}(\vec{u} ; \emptyset)}=\frac{\mathcal{R}_{\vec{Y}}(\vec{u} ; \emptyset)}{\mathcal{R}_{\vec{Y}^{\prime}}(\vec{u} ; \emptyset)} .
$$

This already suffices: Using Eq. (49), we can reduce the statement of the theorem to the case $\vec{Y}=$ $\left(Y_{1}, \ldots, Y_{r-1}, \emptyset\right)$. Both $\mathcal{Z}_{\vec{Y}}(\vec{u} ; \emptyset)$ and $\mathcal{R}_{\vec{Y}}(\vec{u} ; \emptyset)$ are invariant under simultaneous permutation of the components of $\vec{Y}=\left(Y_{1}, \ldots, Y_{r}\right)$ and $u=\left(u_{1}, \ldots, u_{r}\right)$. This follows directly from the respective definitions. Hence, we can reduce the statement of the theorem to the case $\vec{Y}=\left(Y_{1}, \ldots, Y_{r-2}, \emptyset, Y_{r}\right)$ and, again using Eq. (49), to the case $\vec{Y}=\left(Y_{1}, \ldots, Y_{r-2}, \emptyset, \emptyset\right)$. Continuing in this fashion, we can reduce the statement to the case $\vec{Y}=(\emptyset, \ldots, \emptyset)$, in which it holds trivially.

In the calculation of both sides of Eq. (49) we have to evaluate telescopic products. In order to group the factors for such evaluations, we will have to keep track when $Y_{\alpha}(x)$ and $Y_{\alpha}^{T}(y)$ remain constant as we vary the row and column indices. We write

$$
Y_{\alpha}=\left(Y_{\alpha}(1), \ldots, Y_{\alpha}\left(l\left(Y_{\alpha}\right)\right)\right)=(\underbrace{F_{\alpha}(1), \ldots, F_{\alpha}(1)}_{G_{\alpha}(1) \text { times }}, \ldots, \underbrace{F_{\alpha}\left(m_{\alpha}\right), \ldots, F_{\alpha}\left(m_{\alpha}\right)}_{G_{\alpha}\left(m_{\alpha}\right) \text { times }}) .
$$

We set $F_{\alpha}\left(m_{\alpha}+1\right)=0$. Note that for any $x, y \in \mathbb{N}$,

$$
\begin{aligned}
Y_{\alpha}(y) & \in\left\{F_{\alpha}(j): j=1, \ldots, m_{\alpha}+1\right\} \\
Y_{\alpha}^{T}(x) & \in\left\{G_{\alpha}(1)+\cdots+G_{\alpha}(j): j=0, \ldots, m_{\alpha}\right\} .
\end{aligned}
$$

Define the index $j_{\alpha}$ by

$$
G_{\alpha}(1)+\cdots+G_{\alpha}\left(j_{\alpha}-1\right)<l \leq G_{\alpha}(1)+\cdots G_{\alpha}\left(j_{\alpha}\right)
$$


when this condition can be satisfied and $j_{\alpha}=m_{\alpha}+1$ otherwise. We also introduce the notation $H_{\alpha}(j)=$ $G_{\alpha}(1)+\cdots+G_{\alpha}(j)$. We split products over rows of Young diagrams as

$$
\prod_{x=1}^{l}=\prod_{j=1}^{j_{\alpha}-1} \prod_{x=H_{\alpha}(j-1)+1}^{H_{\alpha}(j)} \times \prod_{x=H_{\alpha}\left(j_{\alpha}-1\right)+1}^{l} .
$$

When $x$ comes from the product with index $j \in\left\{1, \ldots, j_{\alpha}-1\right\}$, we have $Y_{\alpha}(y)=F_{\alpha}(j)$. In the remaining product, we have $Y_{\alpha}(y)=F_{\alpha}\left(j_{\alpha}\right)$. Products over columns of Young diagrams are grouped as

$$
\prod_{y=1}^{Y_{\alpha}(l)}=\prod_{y=1}^{F_{\alpha}\left(j_{\alpha}\right)}=\prod_{j=j_{\alpha}}^{m_{\alpha}} \prod_{y=F_{\alpha}(j+1)+1}^{F_{\alpha}(j)}
$$

where we have $Y_{\alpha}^{T}(y)=H_{\alpha}(j)$ if $y$ comes from the factor with value $j$.

The right hand side of Eq. (49) equals

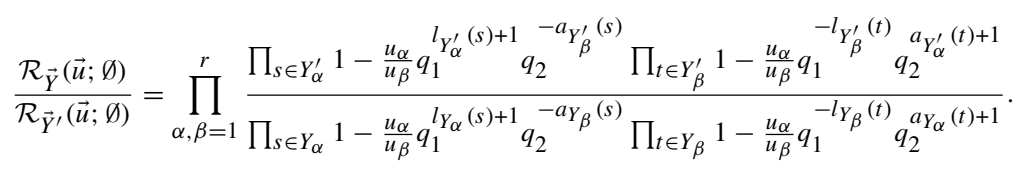

We introduce a variable $\xi$ to be able to ignore poles during the calculation. Regrouping we get

$$
\frac{\mathcal{R}_{\vec{Y}}(\vec{u} ; \emptyset)}{\mathcal{R}_{\vec{Y}^{\prime}}(\vec{u} ; \emptyset)}=\lim _{\xi \rightarrow 1} S(\xi) \prod_{\alpha=1}^{r-1} T_{\alpha}(\xi) U_{\alpha}(\xi)
$$

where

$$
\begin{aligned}
& S(\xi)=\frac{1}{\left(\xi-q_{1}\right)\left(\xi-q_{2}\right)} \prod_{t \in Y_{r}^{\prime}} \frac{\left(\xi-q_{1}^{l_{Y_{r}^{\prime}}(t)+1} q_{2}^{-a_{Y_{r}^{\prime}}(t)}\right)\left(\xi-q_{1}^{-l_{Y_{r}^{\prime}}(t)} q_{2}^{a_{Y_{r}^{\prime}}(t)+1}\right)}{\left(\xi-q_{1}^{l_{Y_{r}}(t)+1} q_{2}^{-a_{Y_{r}}(t)}\right)\left(\xi-q_{1}^{-l_{Y_{r}}(t)} q_{2}^{a_{Y_{r}}(t)+1}\right)} \\
& T_{\alpha}(\xi)=\frac{1}{\xi-u_{\alpha} u_{r}^{-1} q_{1} q_{2}^{a_{Y_{\alpha}}(l, w)+1}} \prod_{t \in Y_{r}} \frac{\xi-u_{\alpha} u_{r}^{-1} q_{1}^{-l_{Y_{r}^{\prime}}(t)} q_{2}^{a_{Y_{\alpha}}(t)+1}}{\xi-u_{\alpha} u_{r}^{-1} q_{1}^{-l_{Y_{r}}(t)} q_{2}^{a_{Y_{\alpha}}(t)+1}} \\
& \times \prod_{s \in Y_{\alpha}} \frac{\xi-u_{\alpha} u_{r}^{-1} q_{1}^{l_{Y_{\alpha}}(s)+1} q_{2}^{-a_{Y_{r}^{\prime}}(s)}}{\xi-u_{\alpha} u_{r}^{-1} q_{1}^{l_{Y_{\alpha}}(s)+1} q_{2}^{-a_{Y_{r}}(s)}} \\
& U_{\alpha}(\xi)=\frac{1}{\xi-u_{r} u_{\alpha}^{-1} q_{2}^{-a_{Y_{\alpha}}(l, w)}} \prod_{t \in Y_{r}} \frac{\xi-u_{r} u_{\alpha}^{-1} q_{1}^{l_{Y_{r}^{\prime}}(t)+1} q_{2}^{-a_{Y_{\alpha}}(t)}}{\xi-u_{r} u_{\alpha}^{-1} q_{1}^{l_{Y_{r}}(t)+1} q_{2}^{-a_{Y_{\alpha}}(t)}} \\
& \times \prod_{s \in Y_{\alpha}} \frac{\xi-u_{r} u_{\alpha}^{-1} q_{1}^{-l_{Y_{\alpha}}(s)} q_{2}^{a_{Y_{r}^{\prime}}(s)+1}}{\xi-u_{r} u_{\alpha}^{-1} q_{1}^{-l_{Y_{\alpha}}(s)} q_{2}^{a_{Y_{r}}(s)+1}} .
\end{aligned}
$$

Using

$$
\begin{gathered}
a_{Y_{\alpha}^{\prime}}(x, y)=\left\{\begin{array}{ll}
Y_{\alpha}(x)-y-1, & x=l, \alpha=r \\
Y_{\alpha}(x)-y, & \text { otherwise }
\end{array},\right. \\
l_{Y_{\alpha}^{\prime}}(x, y)= \begin{cases}Y_{\alpha}^{T}(y)-x-1, & y=w, \alpha=r \\
Y_{\alpha}^{T}(y)-x, & \text { otherwise }\end{cases}
\end{gathered}
$$

and the splitting described in Eqs. (50) and (51) we get

$$
S(\xi)=\frac{1}{\left(\xi-q_{1} q_{2}^{-w+1}\right)\left(\xi-q_{2}^{w}\right)} \frac{\left(\xi-q_{1}\right)\left(\xi-q_{2}\right)}{(\xi-1)\left(\xi-q_{1} q_{2}\right)}
$$




$$
\begin{aligned}
& \prod_{j=1}^{m_{r}} \frac{\left(\xi-q_{1}^{l-H_{r}(j)} q_{2}^{-F_{r}(j)+w}\right)\left(\xi-q_{1}^{-l+H_{r}(j)+1} q_{2}^{F_{r}(j)-w+1}\right)}{\left(\xi-q_{1}^{l-H_{r}(j-1)} q_{2}^{-F_{r}(j)+w}\right)\left(\xi-q_{1}^{-l+H_{r}(j-1)+1} q_{2}^{F_{r}(j)-w+1}\right)}, \\
T_{\alpha}(\xi)= & \frac{1}{\xi-u_{\alpha} u_{r}^{-1} q_{1}^{l\left(Y_{\alpha}\right)-l+1} q_{2}^{-w+1}} \prod_{j=1}^{m_{\alpha}} \frac{\xi-u_{\alpha} u_{r}^{-1} q_{1}^{-l+H_{\alpha}(j)+1} q_{2}^{F_{\alpha}(j)-w+1}}{\xi-u_{\alpha} u_{r}^{-1} q_{1}^{-l+H_{\alpha}(j-1)+1} q_{2}^{F_{\alpha}(j)-w+1}}, \\
U_{\alpha}(\xi)= & \frac{1}{\xi-u_{r} u_{\alpha}^{-1} q_{1}^{-l\left(Y_{\alpha}\right)+l} q_{2}^{w}} \prod_{j=1}^{m_{\alpha}} \frac{\xi-u_{r} u_{\alpha}^{-1} q_{1}^{l-H_{\alpha}(j)} q_{2}^{-F_{\alpha}(j)+w}}{\xi-u_{r} u_{\alpha}^{-1} q_{1}^{l-H_{\alpha}(j-1)} q_{2}^{-F_{\alpha}(j)+w}} .
\end{aligned}
$$

Together

$$
\begin{aligned}
& \frac{\mathcal{R}_{\vec{Y}}(\vec{u} ; \emptyset)}{\mathcal{R}_{\vec{Y}^{\prime}}(\vec{u} ; \emptyset)}=\lim _{\xi \rightarrow 1} \frac{\left(\xi-q_{1}\right)\left(\xi-q_{2}\right)}{(\xi-1)\left(\xi-q_{1} q_{2}\right)} \\
& \quad \prod_{\alpha=1}^{r}\left(\frac{1}{\left(\xi-u_{\alpha} u_{r}^{-1} q_{1}^{l\left(Y_{\alpha}\right)-l+1} q_{2}^{-w+1}\right)\left(\xi-u_{r} u_{\alpha}^{-1} q_{1}^{-l\left(Y_{\alpha}\right)+l} q_{2}^{w}\right)}\right. \\
& \left.\quad \times \prod_{j=1}^{m_{\alpha}} \frac{\left(\xi-u_{r} u_{\alpha}^{-1} q_{1}^{l-H_{\alpha}(j)} q_{2}^{-F_{\alpha}(j)+w}\right)\left(\xi-u_{\alpha} u_{r}^{-1} q_{1}^{-l+H_{\alpha}(j)+1} q_{2}^{F_{\alpha}(j)-w+1}\right)}{\left(\xi-u_{r} u_{\alpha}^{-1} q_{1}^{l-H_{\alpha}(j-1)} q_{2}^{-F_{\alpha}(j)+w}\right)\left(\xi-u_{\alpha} u_{r}^{-1} q_{1}^{-l+H_{\alpha}(j-1)+1} q_{2}^{F_{\alpha}(j)-w+1}\right)}\right) .
\end{aligned}
$$

For the residue calculation, fix the order of the variables such that the integration over $z_{n}$ picks up the residue $z_{l, w}^{r}$ coming from the box $(l, w) \in Y_{r}$ we remove from the last partition in $\vec{Y}$ to get $\vec{Y}^{\prime}$. The left hand side of Eq. (49) equals

$$
\frac{\mathcal{Z}_{\vec{Y}}(\vec{u} ; \emptyset)}{\mathcal{Z}_{\vec{Y}^{\prime}}(\vec{u} ; \emptyset)}=\frac{1-q_{1} q_{2}}{\left(1-q_{1}\right)\left(1-q_{2}\right)} \lim _{z_{j} \rightarrow \hat{z}_{j}, j=1, \ldots, n}\left(1-\frac{z_{l, w}^{r}}{z_{n}}\right) \frac{\mathcal{I}\left(z_{1}, \ldots, z_{n} ; \vec{u}\right)}{\mathcal{I}\left(z_{1}, \ldots, z_{n-1} ; \vec{u}\right)}
$$

We take the first $(n-1)$ limits separately: The quotient $\frac{\mathcal{I}\left(z_{1}, \ldots, z_{n} ; \vec{u}\right)}{\mathcal{I}\left(z_{1}, \ldots, z_{n-1} ; \vec{u}\right)}$ converges to

$$
\begin{aligned}
& \prod_{\alpha=1}^{r}\left(\frac{-u_{\alpha} z_{n}}{\left(z_{n}-u_{\alpha}\right)\left(q_{1} q_{2} z_{n}-u_{\alpha}\right)}\right. \\
& \left.\quad \times \prod_{s \in Y_{\alpha}^{\prime}} \frac{\left(z_{n}-z_{s}^{\alpha}\right)^{2}\left(z_{n}-q_{1} q_{2} z_{s}^{\alpha}\right)\left(z_{n}-q_{1}^{-1} q_{2}^{-1} z_{s}^{\alpha}\right)}{\left(z_{n}-q_{1} z_{s}^{\alpha}\right)\left(z_{n}-q_{2} z_{s}^{\alpha}\right)\left(z_{n}-q_{1}^{-1} z_{s}^{\alpha}\right)\left(z_{n}-q_{2}^{-1} z_{s}^{\alpha}\right)}\right)
\end{aligned}
$$

for $z_{j} \rightarrow \hat{z}_{j}, j=1, \ldots, n-1$. The factors with $\alpha \neq r$ do not have poles for $z_{n} \rightarrow z_{l, w}^{r}$ since $u_{\alpha} / u_{r} \notin$ $\left\{q_{1}^{x} q_{2}^{y}: x, y \in \mathbb{Z}\right\}$. We define $\xi=\frac{z_{n}}{z_{l, w}^{r}}$ and set

$$
A_{\alpha}(\xi):=\prod_{s \in Y_{\alpha}} \frac{\left(\xi-\frac{z_{s}^{\alpha}}{z_{l, w}^{r}}\right)\left(\xi-q_{1} q_{2} \frac{z_{s}^{\alpha}}{z_{l, w}^{r}}\right)\left(\xi-\frac{z_{s}^{\alpha}}{z_{l, w}^{r}}\right)\left(\xi-q_{1}^{-1} q_{2}^{-1} \frac{z_{s}^{\alpha}}{z_{l, w}^{r}}\right)}{\left(\xi-q_{1} \frac{z_{s}^{\alpha}}{z_{l, w}^{r}}\right)\left(\xi-q_{2} \frac{z_{s}^{\alpha}}{z_{l, w}^{r}}\right)\left(\xi-q_{1}^{-1} \frac{z_{s}^{\alpha}}{z_{l, w}^{r}}\right)\left(\xi-q_{2}^{-1} \frac{z_{s}^{\alpha}}{z_{l, w}^{r}}\right)} .
$$

Hence, the remaining limit $z_{n} \rightarrow z_{l, w}^{r}$ is given by

$$
\frac{\mathcal{Z}_{\vec{Y}}(\vec{u} ; \emptyset)}{\mathcal{Z}_{\vec{Y}^{\prime}}(\vec{u} ; \emptyset)}=\lim _{\xi \rightarrow 1} \frac{\left(\xi-q_{1}^{-1}\right)\left(\xi-q_{2}^{-1}\right)}{(\xi-1)\left(\xi-q_{1}^{-1} q_{2}^{-1}\right)} \prod_{\alpha=1}^{r}\left(\frac{-\xi u_{\alpha} z_{l, w}^{r}}{\left(\xi z_{l, w}^{r}-u_{\alpha}\right)\left(q_{1} q_{2} \xi z_{l, w}^{r}-u_{\alpha}\right)} A_{\alpha}(\xi)\right) .
$$

Using the splitting described in (50) and (51) we get

$$
\begin{aligned}
A_{\alpha}(\xi)= & \frac{\left(\xi-\frac{u_{\alpha}}{u_{r}} q_{1}^{1-l} q_{2}^{1-w}\right)\left(\xi-\frac{u_{\alpha}}{u_{r}} q_{1}^{-l} q_{2}^{-w}\right)}{\left(\xi-\frac{u_{\alpha}}{u_{r}} q_{1}^{l\left(Y_{\alpha}\right)-l+1} q_{2}^{1-w}\right)\left(\xi-\frac{u_{\alpha}}{u_{r}} q_{1}^{l\left(Y_{\alpha}\right)-l} q_{2}^{-w}\right)} \\
& \times \prod_{j=1}^{m_{\alpha}} \frac{\left(\xi-\frac{u_{\alpha}}{u_{r}} q_{1}^{H_{\alpha}(j)-l+1} q_{2}^{F_{\alpha}(j)-w+1}\right)\left(\xi-\frac{u_{\alpha}}{u_{r}} q_{1}^{H_{\alpha}(j)-l} q_{2}^{F_{\alpha}(j)-w}\right)}{\left(\xi-\frac{u_{\alpha}}{u_{r}} q_{1}^{H_{\alpha}(j-1)-l+1} q_{2}^{F_{\alpha}(j)-w+1}\right)\left(\xi-\frac{u_{\alpha}}{u_{r}} q_{1}^{H_{\alpha}(j-1)-l} q_{2}^{F_{\alpha}(j)-w}\right)} .
\end{aligned}
$$


Finally, we use $(\rho-\sigma)=-\rho \sigma\left(\rho^{-1}-\sigma^{-1}\right)$ repeatedly to conclude

$$
\begin{aligned}
& \frac{\mathcal{Z}_{\vec{Y}}(\vec{u} ; \emptyset)}{\mathcal{Z}_{\vec{Y}^{\prime}}(\vec{u} ; \emptyset)}=\lim _{\xi \rightarrow 1} \frac{\left(\xi-q_{1}^{-1}\right)\left(\xi-q_{2}^{-1}\right)}{(\xi-1)\left(\xi-q_{1}^{-1} q_{2}^{-1}\right)} \\
& \prod_{\alpha=1}^{r}\left(\frac{-\xi u_{\alpha} u_{r}^{-1} q_{1}^{-l} q_{2}^{-w}}{\left(\xi-\frac{u_{\alpha}}{u_{r}} q_{1}^{l\left(Y_{\alpha}\right)-l+1} q_{2}^{1-w}\right)\left(\xi-\frac{u_{\alpha}}{u_{r}} q_{1}^{l\left(Y_{\alpha}\right)-l} q_{2}^{-w}\right)}\right. \\
& \left.\times \prod_{j=1}^{m_{\alpha}} \frac{\left(\xi-\frac{u_{\alpha}}{u_{r}} q_{1}^{H_{\alpha}(j)-l+1} q_{2}^{F_{\alpha}(j)-w+1}\right)\left(\xi-\frac{u_{\alpha}}{u_{r}} q_{1}^{H_{\alpha}(j)-l} q_{2}^{F_{\alpha}(j)-w}\right)}{\left(\xi-\frac{u_{\alpha}}{u_{r}} q_{1}^{H_{\alpha}(j-1)-l+1} q_{2}^{F_{\alpha}(j)-w+1}\right)\left(\xi-\frac{u_{\alpha}}{u_{r}} q_{1}^{H_{\alpha}(j-1)-l} q_{2}^{F_{\alpha}(j)-w}\right)}\right) \\
& =\lim _{\xi \rightarrow 1} \frac{\left(\xi^{-1}-q_{1}\right)\left(\xi^{-1}-q_{2}\right)}{\left(\xi^{-1}-1\right)\left(\xi^{-1}-q_{1} q_{2}\right)} \\
& \prod_{\alpha=1}^{r}\left(\frac{1}{\left(\xi-\frac{u_{\alpha}}{u_{r}} q_{1}^{l\left(Y_{\alpha}\right)-l+1} q_{2}^{1-w}\right)\left(\xi^{-1}-\frac{u_{r}}{u_{\alpha}} q_{1}^{-l\left(Y_{\alpha}\right)+l} q_{2}^{w}\right)}\right. \\
& \left.\times \prod_{j=1}^{m_{\alpha}} \frac{\left(\xi-\frac{u_{\alpha}}{u_{r}} q_{1}^{H_{\alpha}(j)-l+1} q_{2}^{F_{\alpha}(j)-w+1}\right)\left(\xi^{-1}-\frac{u_{r}}{u_{\alpha}} q_{1}^{-H_{\alpha}(j)+l} q_{2}^{-F_{\alpha}(j)+w}\right)}{\left(\xi-\frac{u_{\alpha}}{u_{r}} q_{1}^{H_{\alpha}(j-1)-l+1} q_{2}^{F_{\alpha}(j)-w+1}\right)\left(\xi^{-1}-\frac{u_{r}}{u_{\alpha}} q_{1}^{-H_{\alpha}(j-1)+l} q_{2}^{-F_{\alpha}(j)+w}\right)}\right) \\
& =\frac{\mathcal{R}_{\vec{Y}}(\vec{u} ; \emptyset)}{\mathcal{R}_{\vec{Y}^{\prime}}(\vec{u} ; \emptyset)}
\end{aligned}
$$

since no factor $\left(\xi^{+1}-\cdots\right)$ vanishes in the limit.

\section{References}

1. Alba, V.A., Fateev, V.A., Litvinov, A.V., Tarnopolskiy, G.M.: On combinatorial expansion of the conformal blocks arising from AGT conjecture. Lett. Math. Phys. 98, 33-64 (2011)

2. Alday, L.F., Gaiotto, D., Tachikawa, Y.: Liouville correlation functions from four-dimensional gauge theories. Lett. Math. Phys. 91, 167-197 (2010)

3. Awata, H., Yamada, Y.: Five-dimensional AGT conjecture and the deformed Virasoro algebra. JHEP 01, 125 (2010)

4. Berest, Y., Felder, G., Patotski, S., Ramadoss, A.C., Willwacher, T.: Representation homology, Lie algebra cohomology and derived Harish-Chandra homomorphism. J. Eur. Math. Soc. 19(9), 2811-2893 (2017)

5. Bershtein, M.A., Shchechkin, A.I.: q-Deformed Painlevé $\tau$ function and q-deformed conformal blocks. J. Phys. A Math. Theor. 50(8), 085202 (2017)

6. Bochner, S., Martin, W.T.: Several Complex Variables. Princeton Mathematical Series, vol. 10. Princeton University Press, Princeton (1948)

7. Chen, H.: Four Ways to Evaluate a Poisson Integral. Math. Mag. 75(4), 290-294 (2002)

8. Fateev, V.A., Litvinov, A.V.: On AGT conjecture. J. High Energy Phys. 2, 14 (2010)

9. Gaiotto, Davide: Asymptotically free $\mathcal{N}=2$ theories and irregular conformal blocks. J. Phys. Conf. Ser. 462(1), 012014 (2013)

10. Hadasz, L., Jaskólski, Z., Suchanek, P.: Proving the AGT relation for $\mathrm{N}_{f}=0,1,2$ antifundamentals. J. High Energy Phys. 6, 46 (2010)

11. Hama, N., Hosomichi, K.: Seiberg-Witten theories on ellipsoids. J. High Energy Phys. 9, 033, front matter+27 (2012). Addendum: JHEP 10, 051 (2012)

12. Its, A., Lisovyy, O., Tykhyy, Y.: Connection problem for the sine-Gordon/Painlevé III tau function and irregular conformal blocks. Int. Math. Res. Not. 18, 8903-8924 (2015)

13. Johansson, K.: On fluctuations of eigenvalues of random hermitian matrices. Duke Math. J. 91(1), 151204, 01 (1998)

14. Moore, G., Nekrasov, N., Shatashvili, S.: Integrating over Higgs branches. Commun. Math. Phys. 209(1), 97-121 (2000)

15. Nakajima, H., Yoshioka, K.: Instanton counting on blowup. 1. Invent. Math. 162, 313-355 (2005)

16. Nakajima, H., Yoshioka, K.: Instanton counting on blowup. II: $K$-theoretic partition function. Transform. Groups 10(3-4), 489-519 (2005) 
17. Negut, A.: Quantum algebras and cyclic quiver varieties. arXiv:1504.06525 (2015)

18. Nekrasov, N.A.: Seiberg-Witten prepotential from instanton counting. Adv. Theor. Math. Phys. 7(5), 831864 (2003)

19. Nekrasov, N.A., Okounkov, A.: Seiberg-Witten theory and random partitions. In: Etingof, P., Retakh, V., Singer, I.M. (eds.) The Unity of Mathematics. Progress in Mathematics, vol. 244, pp. 525-596. Birkhäuser, Boston (2006)

20. Pasquetti, S.: Holomorphic blocks and the 5d AGT correspondence. In: Pestun, V., Zabzine, M. (eds.) Localization Techniques in Quantum Field Theories, chap. 12. Journal of Physics A. arXiv:1608.02968 (2017)

21. Pestun, V.: Localization of gauge theory on a four-sphere and supersymmetric Wilson loops. Commun. Math. Phys. 313(1), 71-129 (2012)

22. Seiberg, N., Witten, E.: Electric-magnetic duality, monopole condensation, and confinement in $N=2$ supersymmetric Yang-Mills theory. Nucl. Phys. B 426(1), 19-52 (1994)

23. Yanagida, S.: Norm of the Whittaker vector of the deformed Virasoro algebra. arXiv:1411.0462 (2014)

24. Yanagida, S.: Five-dimensional SU(2) AGT conjecture and recursive formula of deformed Gaiotto state. J. Math. Phys. 51(12), 123506, 13 (2010)

Communicated by N. Nekrasov 